\title{
Coulomb branch Hilbert series and three dimensional Sicilian theories
}

\author{
Stefano Cremonesi, ${ }^{a}$ Amihay Hanany, ${ }^{a}$ Noppadol Mekareeya ${ }^{b}$ and Alberto Zaffaroni ${ }^{c}, d$ \\ ${ }^{a}$ Theoretical Physics Group, Imperial College London, \\ Prince Consort Road, London, SW7 $2 A Z$ U.K. \\ ${ }^{b}$ Theory Division, Physics Department, CERN, \\ Geneva 23, CH-1211 Switzerland \\ ${ }^{c}$ Dipartimento di Fisica, Università di Milano-Bicocca, \\ Milano, I-20126 Italy \\ ${ }^{d}$ INFN, sezione di Milano-Bicocca, \\ Milano, I-20126 Italy \\ E-mail: s.cremonesi@imperial.ac.uk, a.hanany@imperial.ac.uk, \\ noppadol.mekareeya@cern.ch, alberto.zaffaroni@mib.infn.it
}

ABSTRACT: We evaluate the Coulomb branch Hilbert series of mirrors of three dimensional Sicilian theories, which arise from compactifying the $6 d(2,0)$ theory with symmetry $G$ on a circle times a Riemann surface with punctures. We obtain our result by gluing together the Hilbert series for building blocks $T_{\boldsymbol{\rho}}(G)$, where $\boldsymbol{\rho}$ is a certain partition related to the dual group of $G$, which we evaluated in a previous paper. The result is expressed in terms of a class of symmetric functions, the Hall-Littlewood polynomials. As expected from mirror symmetry, our results agree at genus zero with the superconformal index prediction for the Higgs branch Hilbert series of the Sicilian theories and extend it to higher genus. In the $A_{1}$ case at genus zero, we also evaluate the Coulomb branch Hilbert series of the Sicilian theory itself, showing that it only depends on the number of external legs.

KEYwords: Supersymmetric gauge theory, Supersymmetry and Duality, Duality in Gauge Field Theories

ARXiv EPRINT: 1403.2384 


\section{Contents}

1 Introduction 2

2 Coulomb branch Hilbert series of a $3 \mathrm{~d} \mathcal{N}=4$ gauge theory $\quad 4$

2.1 The monopole formula 4

2.2 The Hall-Littlewood formula 6

$\begin{array}{lll}2.2 .1 & T_{\boldsymbol{\rho}}(\mathrm{SU}(N)) & 6\end{array}$

$\begin{array}{lll}2.2 .2 & T_{\boldsymbol{\rho}}\left(G^{\vee}\right) & 7\end{array}$

3 Mirrors of 3d Sicilian theories of $\boldsymbol{A}$-type $\quad 9$

3.1 Mirrors of tri-vertex theories: star-shaped $\mathrm{U}(2) \times \mathrm{U}(1)^{e} / \mathrm{U}(1)$ quivers $\quad 10$

3.1.1 Computation of the Hilbert series for general $g$ and $e \quad 13$

$\begin{array}{ll}3.2 & \text { The Coulomb branch of the mirror of } T_{N} \\ \end{array}$

3.3 The Coulomb branch of the mirror of a general 3d Sicilian theory 16

$\begin{array}{lll}\text { 3.3.1 The case of genus zero } & 17\end{array}$

3.3.2 Mirror of the SU(3) Sicilian theory with $g=1$ and a maximal puncture 17

4 Mirrors of $3 d$ Sicilian theories of $D$-type $\quad 19$

$\begin{array}{lll}4.1 & D_{3} \text { punctures } & 20\end{array}$

4.1.1 $D_{3}$ punctures: $\left(3^{2}\right),\left(1^{6}\right)$ and $\left(1^{6}\right) \quad 21$

$\begin{array}{lll}4.2 & D_{4} \text { punctures } & 22\end{array}$

4.2.1 $D_{4}$ punctures: $(5,3),\left(2^{2}, 1^{4}\right)$ and $\left(1^{8}\right) \quad 22$

4.2.2 $D_{4}$ punctures: $\left(3^{2}, 1^{2}\right),\left(2^{2}, 1^{4}\right)$ and $\left(2^{2}, 1^{4}\right) \quad 23$

4.2.3 $D_{4}$ punctures: $(5,3),(5,3),\left(2^{4}\right)$ and $\left(3,1^{5}\right) \quad 25$

5 Coulomb branch Hilbert series of $3 d$ theories with tri-vertices 26

$\begin{array}{lll}5.1 & \text { The case of } g=0 & 27\end{array}$

5.1.1 General formula for $g=0$ and any $e \quad 27$

5.1.2 Special case of $g=0$ and $e=6 \quad 28$

5.2 Turning on background fluxes 28

5.3 Generating functions of Coulomb branch Hilbert series 29

5.3.1 Gluing generating functions and recursive formula 30

5.3.2 Proof of the symmetry of the generating functions $G_{e}(\boldsymbol{z}) \quad 32$

6 Conclusion 33

A Mirrors of Sicilian theories with twisted $D$ punctures $\quad 34$

A.1 The Coulomb branch Hilbert series of mirror theories 36

A.1.1 Twisted punctures $\left(2,1^{4}\right),\left(2,1^{4}\right)$ and untwisted puncture $(4,4) \quad 36$

A.1.2 Twisted punctures (6), $\left(1^{6}\right)$ and untwisted puncture $\left(1^{8}\right) \quad 38$

A.1.3 Four $\mathrm{SO}(4)$ twisted punctures: $(2),(2),\left(1^{2}\right),\left(1^{2}\right) \quad 38$ 


\section{Introduction}

A general formula for computing the generating function (Hilbert series) for the chiral ring associated with the Coulomb branch of three dimensional $\mathcal{N}=4$ gauge theories has been recently proposed [1]. The formula counts monopole operators dressed by classical operators and includes quantum corrections. It can be applied to any $3 d \mathcal{N}=4$ supersymmetric gauge theories that possess a Lagrangian description and that are good or ugly in the sense of [2]. The formula has been successfully tested against mirror symmetry in many cases $[1,3]$.

In a companion paper we developed a machinery for computing Coulomb branch Hilbert series for wide classes of $\mathcal{N}=4$ gauge theories by using gluing techniques. We computed the Coulomb branch Hilbert series with background fluxes for the flavor symmetry of the three dimensional superconformal field theories known as $T_{\boldsymbol{\rho}}(G)$ [2], a class of linear quiver theories with non-decreasing ranks associated with a partition $\rho$ and a flavor symmetry $G$. We found an intriguing connection with a class of symmetric functions, the Hall-Littlewood polynomials, which have also appeared in the recent literature in the context of the superconformal index of four dimensional $\mathcal{N}=2$ theories [4]. We clarify the meaning of this connection in the following. The $T_{\boldsymbol{\rho}}(G)$ theories serve as basic building blocks for constructing more complicated theories.

In this paper we consider the theories that arise from compactifying the $6 d(2,0)$ theory with symmetry $G=\mathrm{SU}(N), \mathrm{SO}(2 N)$ on a circle times a Riemann surface with punctures. These are known as three dimensional Sicilian theories. With the exception of the SU(2) case, they have no Lagrangian description [5]. We are interested in their mirror which can be obtained as follows. Starting from a set of building blocks $\left\{T_{\boldsymbol{\rho}_{1}}(G), T_{\boldsymbol{\rho}_{2}}(G), \ldots, T_{\boldsymbol{\rho}_{n}}(G)\right\}$, one can construct a new theory by gauging the common centerless flavor symmetry $G / Z(G)$, where $Z(G)$ is the center of $G$. We refer to this procedure as 'gluing' the building blocks together. The resulting theory is the aforementioned mirror of the theory associated to a sphere with punctures $\left\{\boldsymbol{\rho}_{1}, \boldsymbol{\rho}_{2}, \ldots, \boldsymbol{\rho}_{n}\right\}[6,7]$.

The main purpose of this paper is to compute the Coulomb branch Hilbert series of these mirrors. We do this by gluing together the Hilbert series of the theories $T_{\boldsymbol{\rho}_{i}}(G)$ as explained in [3]. By mirror symmetry, our results should agree with the Higgs branch Hilbert series of the Sicilian theories. The latter can be computed for the four dimensional version of the theory, since the Higgs branch of a theory with eight supercharges is protected against quantum corrections by a non-renormalization theorem [8] and therefore is the same in all dimensions. Although the theory is non-Lagrangian, at genus zero the Higgs branch Hilbert series can be written in terms of the Hall-Littlewood indices proposed in [4, 9]. We find perfect agreement with the results in $[4,9,10]$, as predicted by mirror symmetry.

Our result clarifies why the Hall-Littlewood polynomials appear in two different contexts, the Coulomb branch Hilbert series for the $T_{\boldsymbol{\rho}}(G)$ theories and a limit of the four dimensional superconformal index of Sicilian theories. It is interesting to observe how the structure of the superconformal index formula (see for example (3.31)), obtained in a completely different manner, can be naturally reinterpreted in terms of gluing of three dimensional building blocks. 
Our gluing formula easily extends to punctured Riemann surfaces of higher genus, by incorporating adjoint hypermultiplets in the mirror theory [6]. For such Riemann surfaces the Hall-Littlewood index of the 4d non-Lagrangian theory differs from the Higgs branch Hilbert series, as discussed in [4]. Our formula for the Coulomb branch Hilbert series of the 3d Lagrangian mirror theory (3.30) provides the Higgs branch Hilbert series of the $(3 \mathrm{~d}$ or 4d) non-Lagrangian Sicilian theory, for any genus $g$, as long as the theory is not bad in the sense of [2]. In section 3.1, we successfully test our higher genus prediction for the case of $A_{1}$ Sicilian theories, also known as tri-vertex theories. These are $3 d \mathcal{N}=4$ Lagrangian theories associated to a graph with tri-valent vertices, where a finite line denotes an $\mathrm{SU}(2)$ gauge group, an infinite line denotes an $\mathrm{SU}(2)$ global symmetry, and a vertex denotes 8 half-hypermultiplets in the tri-fundamental representation of $\mathrm{SU}(2)^{3}$. These graphs are characterized by the genus $g$ and the number of external legs $e$. The Higgs branch Hilbert series of such theories were computed directly in [11]. In section 3.1.1 we reproduce that result from the Coulomb branch of the mirror theory.

As an addition to the main line of this paper, which focusses on the Coulomb branch of mirrors of three dimensional Sicilian theories, in section 5 we study the Coulomb branch of tri-vertex theories themselves at genus zero using the monopole formula. We find that the Coulomb branch Hilbert series depends only on $e$ and not on the details of the graph, as suggested in [6].

The paper is organized as follows. In section 2 we review the formula for the Coulomb branch Hilbert series of $\mathcal{N}=4$ theories, the gluing technique and the Hall-Littlewood formula for the $T_{\boldsymbol{\rho}}(G)$ theories. In section 3 we compute the Coulomb branch Hilbert series of the mirrors of three dimensional Sicilian theories with A-type punctures at arbitrary genus $g$. We examine in particular the case of the $T_{N}$ theory. We successfully compare the result for genus zero with the superconformal index prediction for Higgs branch Hilbert series of the Sicilian theories given in [4,9]. We also give explicit examples for theories at higher genus. In the $\mathrm{SU}(2)$ case, where the Sicilian theories are Lagrangian, we compare our result with the Higgs branch Hilbert series computed in [11] finding perfect agreement. In section 4 we extend the analysis to theories of type D. As a general check of our predictions, we demonstrate the equivalence between $D_{3}$ and $A_{3}$ punctures and we compute the Coulomb branch Hilbert series for a set of $D_{4}$ punctures where the Higgs branch Hilbert series can be explicitly evaluated, finding perfect agreement. In section 5 we compute the Hilbert series of the tri-vertex theories at genus zero showing that they only depend on the number of external legs. In section 5.3 we present generating functions and recursive formulae, which are powerful tools for computing the Hilbert series of tri-vertex theories. Finally, in appendix A we consider theories of type D with twisted punctures.

Note added. One might ask whether there is any relation between the Coulomb branch Hilbert series that we study and the $3 d$ superconformal index [12-14]. Indeed, a recent work [15] appeared after the submission of this paper, showing that the superconformal index of a $3 d \mathcal{N}=4$ theory reduces to the Hilbert series in a particular limit. 


\section{Coulomb branch Hilbert series of a $3 \mathrm{~d} \mathcal{N}=4$ gauge theory}

Our main aim is to study the Coulomb branch of three dimensional $\mathcal{N}=4$ gauge theories. Classically, this branch is parameterized by the vacuum expectation values of the triplet of scalars in the $\mathcal{N}=4$ vector multiplets and by the vacuum expectation value of the dual photons, at a generic point where the gauge group is spontaneously broken to its maximal torus. This yields a HyperKähler space of quaternionic dimension equal to the rank of the gauge group. The Coulomb branch is, however, not protected against quantum corrections and the associated chiral ring has a complicated structure involving monopole operators in addition to the classical fields in the Lagrangian.

A suitable quantum description of the chiral ring on the Coulomb branch is to replace the above description by monopole operators. The gauge invariant BPS objects on the branch are monopole operators dressed by a product of a certain scalar field in the vector multiplet. The spectrum of such BPS objects can be studied in a systematic way by computing their partition function, known as the Hilbert series. A Hilbert series is a generating function of the chiral ring, which enumerates gauge invariant BPS operators which have a non-zero expectation value along the Coulomb branch. As extensively discussed in $[1,3]$, a general formula for the Hilbert series of the Coulomb branch of an $\mathcal{N}=4$ theory can be computed based on this principle. We refer to such a formula as the monopole formula.

In [3] we found an analytic expression for the Coulomb branch Hilbert series of a class of theories called $T_{\boldsymbol{\rho}}(G)$ [2], where $G$ is a classical group and $\boldsymbol{\rho}$ is a partition associated with the GNO dual group $G^{\vee}$. Such a theory has a Lagrangian description $[2,3,6]$. The Hilbert series of these theories can be conveniently written in terms of Hall-Littlewood polynomials [3], and the corresponding formula is dubbed the Hall-Littlewood formula. In the following section we show that the Hall-Littlewood formula is a convenient tool to compute the Coulomb branch Hilbert series of mirrors of three dimensional Sicilian theories.

Let us now summarize important information on the monopole and Hall-Littlewood formulae for Coulomb branch Hilbert series.

\subsection{The monopole formula}

The monopole formula [1] counts all gauge invariant chiral operators that can acquire a non-zero expectation value along the Coulomb branch, according to their dimension and quantum numbers. The operators are written in an $\mathcal{N}=2$ formulation and the $\mathcal{N}=4$ vector multiplet is decomposed into an $\mathcal{N}=2$ vector multiplet and a chiral multiplet $\Phi$ transforming in the adjoint representation of the gauge group. We refer to [1] for an explanation of the formula and simply quote the final result here.

The formula for a good or ugly [2] theory with gauge group $G$ reads

$$
H_{G}(t, z)=\sum_{\boldsymbol{m} \in \Gamma_{G} \vee / W_{G} \vee} z^{J(\boldsymbol{m})} t^{\Delta(\boldsymbol{m})} P_{G}(t ; \boldsymbol{m}) .
$$

The sum is over the magnetic charges of the monopoles $m$ which, up to a gauge transformation, belong to a Weyl Chamber of the weight lattice $\Gamma_{G^{\vee}}$ of the GNO dual group [16]. 
$P_{G}(t ; m)$ is a factor which counts the gauge invariants of the gauge group $H_{m}$ unbroken by the monopole $m$ made with the adjoint scalar field $\phi$ in the multiplet $\Phi$, according to their dimension. It is given by

$$
P_{G}(t ; \boldsymbol{m})=\prod_{i=1}^{r} \frac{1}{1-t^{d_{i}(\boldsymbol{m})}},
$$

where $d_{i}(\boldsymbol{m}), i=1, \ldots, \operatorname{rank} H_{\boldsymbol{m}}$ are the degrees of the independent Casimir invariants of $H_{\boldsymbol{m}} . \Delta(m)$ is the quantum dimension of the monopole which is given by [2, 17-19]

$$
\Delta(\boldsymbol{m})=-\sum_{\boldsymbol{\alpha} \in \Delta_{+}(G)}|\alpha(\boldsymbol{m})|+\frac{1}{2} \sum_{i=1}^{n} \sum_{\boldsymbol{\rho}_{i} \in R_{i}}\left|\boldsymbol{\rho}_{i}(\boldsymbol{m})\right|,
$$

where $\boldsymbol{\alpha}$ are the positive roots of $G$ and $\boldsymbol{\rho}_{i} \in R_{i}$ the weights of the matter field representation $R_{i}$ under the gauge group. $z$ is a fugacity valued in the topological symmetry group, which exists if $G$ is not simply connected, and $J(\boldsymbol{m})$ the topological charge of a monopole operator of GNO charges $\boldsymbol{m}$.

Turning on background magnetic fluxes. As discussed in [3], the formula can be generalized to include background monopole fluxes for a global flavor symmetry $G_{F}$ acting on the matter fields:

$$
H_{G, G_{F}}\left(t, \boldsymbol{m}_{F}, z\right)=\sum_{\boldsymbol{m} \in \Gamma_{G} \vee / W_{G} \vee} z^{J(\boldsymbol{m})} t^{\Delta\left(\boldsymbol{m}, \boldsymbol{m}_{\boldsymbol{F}}\right)} P_{G}(t ; \boldsymbol{m}) .
$$

The sum is only over the magnetic fluxes of the gauge group $G$ but depends on the weights $\boldsymbol{m}_{F}$ of the dual group $G_{F}^{\vee}$ which enter explicitly in the dimension formula (2.3) through all the matter fields that are charged under the global symmetry $G_{F}$. By using the global symmetry we can restrict the value of $\boldsymbol{m}_{F}$ to a Weyl chamber of $G_{F}^{\vee}$ and take $\boldsymbol{m}_{F} \in \Gamma_{G_{F}^{\vee}} / W_{G_{F}^{\vee}}$.

The gluing technique. We can construct more complicated theories by starting with a collection of theories and gauging some common global symmetry $G_{F}$ they share. The Hilbert series of the final theory where $G_{F}$ is gauged is given by multiplying the Hilbert series with background fluxes for $G_{F}$ of the building blocks, summing over the monopoles of $G_{F}$ and including the contribution to the dimension formula of the $\mathcal{N}=4$ dynamical vector multiplets associated with the gauged group $G_{F}$ :

$$
H(t)=\sum_{\boldsymbol{m}_{F} \in \Gamma_{G_{F}} / W_{G_{F}}} t^{-\sum_{\boldsymbol{\alpha}_{F} \in \Delta_{+}\left(G_{F}\right)}\left|\alpha_{F}\left(\boldsymbol{m}_{F}\right)\right|} P_{G_{F}}\left(t ; \boldsymbol{m}_{F}\right) \prod_{i} H_{G, G_{F}}^{(i)}\left(t, \boldsymbol{m}_{F}\right),
$$

where $\alpha_{F}$ are the positive roots of $G_{F}$ and the product with the index $i$ runs over the Hilbert series of the $i$-th theory that is taken into the gluing procedure. Since we can always make $\alpha_{F}\left(\boldsymbol{m}_{F}\right)$ non-negative by choosing $\boldsymbol{m}_{F}$ in the main Weyl chamber, the evaluation of $H(t)$ turns out to have no absolute values. The formula (2.5) can be immediately generalized to include fugacities for the topological symmetries acting on the Coulomb branch.

In the next sections we will provide explicit and general formulae for many interesting $3 \mathrm{~d} \mathcal{N}=4$ superconformal theories including mirrors of M5-brane theories compactified on a circle times a Riemann surfaces with punctures. They are obtained by gluing a simple class of building blocks that we now discuss. 


\subsection{The Hall-Littlewood formula}

As extensively discussed in [3], the Coulomb branch Hilbert series of $T_{\boldsymbol{\rho}}\left(G^{\vee}\right)$ for a classical group $G$ can be computed using formulae involving Hall-Littlewood polynomials. The main purpose of this paper is to show that these formulae are useful for computing Coulomb branch Hilbert series of mirrors of $3 d$ Sicilian theories. For the sake of completeness of the paper, we review Hall-Littlewood formulae below. We first present the formula for $G=\mathrm{SU}(N)$ and then discuss the formula for other classical groups, namely $\mathrm{SO}(N)$ and $\operatorname{USp}(2 N)$.

\subsection{1 $T_{\rho}(\mathrm{SU}(N))$}

The quiver diagram for $T_{\boldsymbol{\rho}}(\mathrm{SU}(N))$ is

$$
[\mathrm{U}(N)]-\left(\mathrm{U}\left(N_{1}\right)\right)-\left(\mathrm{U}\left(N_{2}\right)\right)-\cdots-\left(\mathrm{U}\left(N_{d}\right)\right),
$$

where the partition $\boldsymbol{\rho}$ of $N$ is given by

$$
\boldsymbol{\rho}=\left(N-N_{1}, N_{1}-N_{2}, N_{2}-N_{3}, \ldots, N_{d-1}-N_{d}, N_{d}\right),
$$

with the restriction that $\rho$ is a non-increasing sequence:

$$
N-N_{1} \geq N_{1}-N_{2} \geq N_{2}-N_{3} \geq \cdots \geq N_{d-1}-N_{d} \geq N_{d}>0 .
$$

The quiver theory in (2.6) can be realised from brane configurations as proposed in [20].

The Coulomb branch Hilbert series of this theory can be written as

$$
\begin{aligned}
& H\left[T_{\rho}(\mathrm{SU}(N))\right]\left(t ; x_{1}, \ldots, x_{d+1} ; n_{1}, \ldots, n_{N}\right) \\
& \quad=t^{\frac{1}{2} \delta_{\mathrm{U}(N)}(\boldsymbol{n})}(1-t)^{N} K_{\boldsymbol{\rho}}^{\mathrm{U}(N)}(\boldsymbol{x} ; t) \Psi_{\mathrm{U}(N)}^{\boldsymbol{n}}\left(\boldsymbol{x} t^{\frac{1}{2} \boldsymbol{w}_{\boldsymbol{\rho}}} ; t\right),
\end{aligned}
$$

where the Hall-Littlewood polynomial associated with the group $\mathrm{U}(N)$ is given by

$$
\Psi_{\mathrm{U}(N)}^{n}\left(x_{1}, \ldots, x_{N} ; t\right)=\sum_{\sigma \in S_{N}} x_{\sigma(1)}^{n_{1}} \ldots x_{\sigma(N)}^{n_{N}} \prod_{1 \leq i<j \leq N} \frac{1-t x_{\sigma(i)}^{-1} x_{\sigma(j)}}{1-x_{\sigma(i)}^{-1} x_{\sigma(j)}},
$$

with $n_{1}, \ldots, n_{N}$ the background GNO charges for $\mathrm{U}(N)$ group, with

$$
n_{1} \geq n_{2} \geq \cdots \geq n_{N} \geq 0 .
$$

The notation $\delta_{\mathrm{U}(N)}$ denotes the sum over positive roots of the group $\mathrm{U}(N)$ acting on the background charges $n_{i}$ :

$$
\delta_{\mathrm{U}(N)}(\boldsymbol{n})=\sum_{1 \leq i<j \leq N}\left(n_{i}-n_{j}\right)=\sum_{j=1}^{N}(N+1-2 j) n_{j} .
$$

The fugacities $x_{1}, \ldots, x_{d+1}$ are subject to the following constraint which fixes the overall U(1):

$$
\prod_{i=1}^{d+1} x_{i}^{\rho_{i}}=1
$$


The vector $\boldsymbol{w}_{r}$ denotes the weights of the $\mathrm{SU}(2)$ representation of dimension $r$ :

$$
\boldsymbol{w}_{r}=(r-1, r-3, \ldots, 3-r, 1-r) .
$$

Hence the notation $t^{\frac{1}{2} \boldsymbol{w}_{r}}$ represents the vector

$$
t^{\frac{1}{2} \boldsymbol{w}_{r}}=\left(t^{\frac{1}{2}(r-1)}, t^{\frac{1}{2}(r-3)}, \ldots, t^{-\frac{1}{2}(r-3)}, t^{-\frac{1}{2}(r-1)}\right) .
$$

In (2.9) and henceforth, we abbreviate

$$
\Psi_{\mathrm{U}(N)}^{n}\left(\boldsymbol{x} t^{\frac{1}{2} \boldsymbol{w}_{\rho}} ; t\right):=\Psi_{\mathrm{U}(N)}^{\left(n_{1}, \ldots, n_{N}\right)}\left(x_{1} t^{\frac{1}{2} \boldsymbol{w}_{\rho_{1}}}, x_{2} t^{\frac{1}{2} \boldsymbol{w}_{\rho_{2}}}, \ldots, x_{d+1} t^{t^{\frac{1}{2}} \boldsymbol{w}_{\rho_{d+1}}} ; t\right) .
$$

The prefactor $K_{\boldsymbol{\rho}}^{\mathrm{U}(N)}(\boldsymbol{x} ; t)$ is given by

$$
K_{\boldsymbol{\rho}}^{\mathrm{U}(N)}(\boldsymbol{x} ; t)=\prod_{i=1}^{\operatorname{length}\left(\boldsymbol{\rho}^{T}\right)} \prod_{j, k=1}^{\rho_{i}^{T}} \frac{1}{1-a_{j}^{i} \bar{a}_{k}^{i}},
$$

where $\rho^{T}$ denotes the transpose of the partition $\rho$ and we associate the factors

$$
\begin{aligned}
a_{j}^{i} & =x_{j} t^{\frac{1}{2}\left(\rho_{j}-i+1\right)}, & & i=1, \ldots, \rho_{j} \\
\bar{a}_{k}^{i} & =x_{k}^{-1} t^{\frac{1}{2}\left(\rho_{k}-i+1\right)}, & & i=1, \ldots, \rho_{k}
\end{aligned}
$$

to each box in the Young tableau. The powers of $t$ inside $a_{j}^{i}$ and $\bar{a}_{k}^{i}$ are positive by construction.

We demonstrate the HL formula (2.9) in a number of examples in section 3.

\subsection{2 $T_{\rho}\left(G^{\vee}\right)$}

In this section we review a generalized version of formula (2.9) to a more general classical group $G$. The quiver diagrams are explicitly given in [3]. Further discussions regarding mathematical aspects of this formula can be found in [3, 21, 22].

The partition $\boldsymbol{\rho}$ induces an embedding $\boldsymbol{\rho}: \operatorname{Lie}(\mathrm{SU}(2)) \rightarrow \operatorname{Lie}(G)$ such that

$$
[1,0, \ldots, 0]_{G}=\bigoplus_{i}\left[\rho_{i}-1\right]_{\mathrm{SU}(2)}
$$

The global symmetry $G_{\boldsymbol{\rho}}$ associated to the puncture $\boldsymbol{\rho}=\left[\rho_{i}\right]$, with $r_{k}$ the number of times that part $k$ appears in the partition $\boldsymbol{\rho}$, is given by

$$
G_{\rho}= \begin{cases}S\left(\prod_{k} \mathrm{U}\left(r_{k}\right)\right) & G=\mathrm{U}(N), \\ \prod_{k \text { odd }} \mathrm{SO}\left(r_{k}\right) \times \prod_{k \text { even }} \mathrm{USp}\left(r_{k}\right) & G=\mathrm{SO}(2 N+1) \text { or } \mathrm{SO}(2 N), \\ \prod_{k \text { odd }} \mathrm{USp}\left(r_{k}\right) \times \prod_{k \text { even }} \mathrm{SO}\left(r_{k}\right) & G=\mathrm{USp}(2 N) .\end{cases}
$$

Let $x_{1}, x_{2}, \ldots$ be fugacities for the global symmetry $G_{\boldsymbol{\rho}}$, the commutant of $\rho(\mathrm{SU}(2))$ in $G$, and $r(G)$ the rank of $G$. In [3] we have conjectured that the Coulomb branch Hilbert series is given by the HL formula

$$
H\left[T_{\boldsymbol{\rho}}\left(G^{\vee}\right)\right]\left(t ; \boldsymbol{x} ; n_{1}, \ldots, n_{r(G)}\right)=t^{\frac{1}{2} \delta_{G \vee}(\boldsymbol{n})}(1-t)^{r(G)} K_{\boldsymbol{\rho}}^{G}(\boldsymbol{x} ; t) \Psi_{G}^{\boldsymbol{n}}(\boldsymbol{a}(t, \boldsymbol{x}) ; t) .
$$


Here $\Psi_{G}^{n}$ is the Hall-Littlewood polynomial associated to a Lie group $G$, given by

$$
\Psi_{G}^{\boldsymbol{n}}\left(x_{1}, \ldots, x_{r} ; t\right)=\sum_{w \in W_{G}} \boldsymbol{x}^{w(\boldsymbol{n})} \prod_{\boldsymbol{\alpha} \in \Delta_{+}(G)} \frac{1-t \boldsymbol{x}^{-w(\boldsymbol{\alpha})}}{1-\boldsymbol{x}^{-w(\boldsymbol{\alpha})}},
$$

where $W_{G}$ denotes the Weyl group of $G, \Delta_{+}(G)$ the set of positive roots of $G, \boldsymbol{n}=$ $\sum_{i=1}^{r} n_{i} \boldsymbol{e}_{i}$, with $\left\{\boldsymbol{e}_{1}, \ldots, \boldsymbol{e}_{r}\right\}$ the standard basis of the weight lattice and $r$ the rank of $G$. See appendix B of [3] for more details. $G^{\vee}$ is the GNO dual group [16]. The power $\delta_{G^{\vee}}(\boldsymbol{n})$ is the sum over positive roots $\boldsymbol{\alpha} \in \Delta_{+}\left(G^{\vee}\right)$ of the flavor group $G^{\vee}$ acting on the background monopole charges $\boldsymbol{n}$ :

$$
\delta_{G^{\vee}}(\boldsymbol{n})=\sum_{\boldsymbol{\alpha} \in \Delta_{+}\left(G^{\vee}\right)}|\boldsymbol{\alpha}(\boldsymbol{n})|
$$

Explicitly, for classical groups $G$ and fluxes $\boldsymbol{n}$ in the fundamental Weyl chamber, these are given by

$$
\delta_{G^{\vee}}(\boldsymbol{n})= \begin{cases}\sum_{j=1}^{N}(N+1-2 j) n_{j} & G^{\vee}=G=\mathrm{U}(N), \\ \sum_{j=1}^{N}(2 N+1-2 j) n_{j} & G^{\vee}=B_{N}, \quad G=C_{N} \\ \sum_{j=1}^{N}(2 N+2-2 j) n_{j} & G^{\vee}=C_{N}, \quad G=B_{N} \\ \sum_{j=1}^{N-1}(2 N-2 j) n_{j} & G^{\vee}=G=D_{N} .\end{cases}
$$

The argument $\boldsymbol{a}(t, \boldsymbol{x})$ of the HL polynomial, which we shall henceforth abbreviate as $\boldsymbol{a}$, is determined by the following decomposition of the fundamental representation of $G$ to $G_{\rho} \times \rho(\mathrm{SU}(2)):$

$$
\chi_{\text {fund }}^{G}(\boldsymbol{a})=\sum_{k} \chi_{\text {fund }}^{G_{\rho_{k}}}\left(\boldsymbol{x}_{k}\right) \chi_{\left[\rho_{k}-1\right]}^{\mathrm{SU}(2)}\left(t^{1 / 2}\right),
$$

where $G_{\rho_{k}}$ denotes a subgroup of $G_{\boldsymbol{\rho}}$ corresponding to the part $k$ of the partition $\boldsymbol{\rho}$ that appears $r_{k}$ times. Formula (2.25) determines $\boldsymbol{a}$ as a function of $t$ and $\left\{\boldsymbol{x}_{k}\right\}$ as required. Of course, there are many possible choices for $\boldsymbol{a}$; the choices that are related to each other by outer-automorphisms of $G$ are equivalent.

The prefactor $K_{\boldsymbol{\rho}}^{G}(\boldsymbol{x} ; t)$ is independent of $\boldsymbol{n}$ and can be determined as follows. The embedding specified by $\boldsymbol{\rho}$ induces the decomposition

$$
\chi_{\mathbf{A d j}}^{G}(\boldsymbol{a})=\sum_{j=0, \frac{1}{2}, 1, \frac{3}{2}, \ldots} \chi_{R_{j}}^{G_{\boldsymbol{\rho}}}\left(\boldsymbol{x}_{j}\right) \chi_{[2 j]}^{\mathrm{SU}(2)}\left(t^{1 / 2}\right),
$$

where $\boldsymbol{a}$ on the left hand side is the same $\boldsymbol{a}$ as in (2.25). Each term in the previous formula gives rise to a plethystic exponential, ${ }^{1}$ giving

$$
K_{\boldsymbol{\rho}}^{G}(\boldsymbol{x} ; t)=\mathrm{PE}\left[\sum_{j=0, \frac{1}{2}, 1, \frac{3}{2}, \ldots} t^{j+1} \chi_{R_{j}}^{G_{\boldsymbol{\rho}}}\left(\boldsymbol{x}_{j}\right)\right] .
$$

\footnotetext{
${ }^{1}$ See appendix A of [1] for the definition.
} 
As a remark, in the special case when $\rho: \operatorname{Lie}(\mathrm{SU}(2)) \rightarrow \operatorname{Lie}(G)$ is a principal embedding $\boldsymbol{\rho}_{\text {princ }}$ (see, e.g. [22]), the global symmetry acting on the Coulomb branch is trivial $G_{\boldsymbol{\rho}_{\text {princ }}}=$ 1 , the prefactor $K_{\boldsymbol{\rho}_{\text {princ }}}^{G}(t)=P_{G}(t ; \mathbf{0})$ equals the Casimir factor of $G$ (or equivalently $G^{\vee}$ ), and the Hall-Littlewood formula (2.21) reduces to

$$
H\left[T_{\boldsymbol{\rho}_{\text {princ }}}\left(G^{\vee}\right)\right]\left(t ; n_{1}, \ldots, n_{r(G)}\right)=1 .
$$

This identity has a simple physical interpretation in the context of mirrors of Sicilian theories that we consider in this paper: adding an empty puncture does not affect the Hilbert series of the Sicilian theory.

In the following we discuss several examples of mirrors of $3 d$ Sicilian theories for which we use the Hall-Littlewood formulae to compute their Coulomb branch Hilbert series.

\section{Mirrors of 3d Sicilian theories of $A$-type}

In this and the next section we evaluate the Coulomb branch Hilbert series of the mirror of the theories arising from compactifying the $6 d(2,0)$ theory with symmetry $G$ on a circle times a Riemann surface with punctures, also called Sicilian theories. These theories and their Coulomb branch Hilbert series will be obtained by gluing together $T_{\boldsymbol{\rho}}(G)$ building blocks.

Given a set of theories $\left\{T_{\boldsymbol{\rho}_{1}}(G), \ldots, T_{\boldsymbol{\rho}_{n}}(G)\right\}$, we can construct a new theory by gauging the common centerless flavor symmetry $G / Z(G)$; see figure $1 .^{2}$ The resulting theory is the mirror of the theory on M5-branes wrapping a circle times a Riemann sphere with punctures $\boldsymbol{\rho}_{1}, \ldots, \boldsymbol{\rho}_{n}[6,7]$. For example, taking $G=\mathrm{SU}(3)$ and $\boldsymbol{\rho}_{1}=\boldsymbol{\rho}_{2}=\boldsymbol{\rho}_{3}=(1,1,1)$ we obtain a mirror of the $T_{3}$ theory reduced to three dimensions. Recall that the Higgs branch of the $3 \mathrm{~d} T_{3}$ theory is the reduced moduli space of $1 E_{6}$ instantons on $\mathbb{C}^{2}$ and the Coulomb branch is $\mathbb{C}^{2} / \widehat{E}_{6}$. The moduli spaces of $k E_{6}, E_{7}$ and $E_{8}$ instantons on $\mathbb{C}^{2}$ can be also realized as the Higgs branch of the $6 d(2,0)$ theory compactified on a circle times a Riemann sphere with punctures.

We demonstrate how to 'glue' the Hilbert series $T_{\boldsymbol{\rho}_{n}}(G)$ together to obtain the Coulomb branch Hilbert series of the mirror of the theory on M5-branes compactified on $S^{1}$ times a Riemann sphere with punctures $\left\{\boldsymbol{\rho}_{i}\right\}$. By mirror symmetry, this is equal to the Higgs branch Hilbert series of the latter. The theories on M5-branes are not Lagrangian, but, when the genus of the Riemann surface is zero, the Higgs branch Hilbert series can be evaluated by the Hall-Littlewood (HL) limit of the superconformal index [4]. We find perfect agreement with the results in [4], which were obtained in a completely different manner. Upon introduction of $g G$-adjoint hypermultiplets [6], our formulae can be used also for genus greater than one, where the Higgs branch Hilbert series for the M5-brane theories cannot be evaluated as a limit of the $4 d$ superconformal index. ${ }^{3}$ In section 3.1 we will be able to test the validity of our result for higher genus in the case of two M5 branes

\footnotetext{
${ }^{2}$ For $G=\mathrm{U}(N)$, this also involves factoring out a decoupled $\mathrm{U}(1)$ gauge group.

${ }^{3}$ For genus greater than 1 , the F-terms of the theory are not all independent. As a result, the HL limit of the $4 d$ superconformal index fails to reproduce the Higgs branch Hilbert series. For a very clear explanation of this technical fact, see section 5 of [4].
} 




Figure 1. Gluing $T_{\boldsymbol{\rho}_{1}}(G), \ldots, T_{\boldsymbol{\rho}_{n}}(G)$ via the common centerless flavor symmetry $G / Z(G)$. This is a mirror theory of the theory on M5-brane compactified on a circle times a Riemann sphere with punctures $\boldsymbol{\rho}_{1}, \ldots, \boldsymbol{\rho}_{n}$.

where the theory is Lagrangian and we can use conventional methods for computing the Higgs branch Hilbert series.

In this section we discuss the case of A-type theories with $G=\mathrm{SU}(N)$ and in the next section we discuss D-type theories with $G=\mathrm{SO}(2 N)$.

\subsection{Mirrors of tri-vertex theories: star-shaped $U(2) \times U(1)^{e} / U(1)$ quivers}

We start by considering the Coulomb branch Hilbert series of the mirrors of theories on two M5-branes compactified on a circle times a Riemann surface with punctures. The latter are referred to as $3 d \mathrm{SU}(2)$ Sicilian theories [6, 23] or $3 d$ theories with tri-vertices [11]. They are Lagrangian theories whose quiver is explicitly discussed in section 5. According to [6], the mirror of a tri-vertex theory with genus $g$ and $e$ external legs is a star-shaped $\mathrm{U}(2) \times \mathrm{U}(1)^{e} / \mathrm{U}(1)$ quiver gauge theory with the $\mathrm{U}(2)$ node with $g$ adjoint hypermultiplets in the center, attached to $e \geq 3 \mathrm{U}(1)$ nodes around it. The quiver is depicted in figure 2.

The overall $\mathrm{U}(1)$ gauge group in the quiver is decoupled and needs to be factored out. It is crucial to mod out by the overall $\mathrm{U}(1)$ properly: in particular the quiver with an $\mathrm{SU}(2)$ node in the center, attached to $e \mathrm{U}(1)$ nodes around it, gives the wrong Coulomb branch, which disagrees with the Higgs branch of the $g=0$ tri-vertex theory with $e \geq 3$ legs [11]. The reason is that $\mathrm{U}(2)=\mathrm{U}(1) \times \mathrm{SU}(2) / \mathbb{Z}_{2}$.

Let us first consider the $\mathrm{U}(2) \times \mathrm{U}(1)^{e}$ quiver gauge theory which includes the decoupled overall $\mathrm{U}(1)$. We use GNO charges $n_{1}$ and $n_{2}$ for $\mathrm{U}(2)$, related to the integer weights $n_{1} \geq n_{2}>-\infty$ in the Weyl chamber. For the $i$-th $\mathrm{U}(1)$ gauge group, with $i=1, \ldots, e$, we 


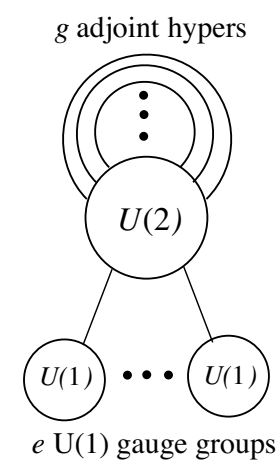

Factoring out by the overall U(1)

Figure 2. The mirror theory of a tri-vertex theory with genus $g$ and $e$ external legs.

use the GNO charge $m_{i} \in \mathbb{Z}$. The dimension formula (2.3) reads

$$
\Delta_{g}\left(n_{1}, n_{2} ; m_{1}, \ldots, m_{e}\right)=(g-1)\left|n_{1}-n_{2}\right|+\frac{1}{2} \sum_{i=1}^{e}\left(\left|n_{1}-m_{i}\right|+\left|n_{2}-m_{i}\right|\right) .
$$

The formula is invariant under the common shift $n_{1,2} \rightarrow n_{1,2}+c, m_{i} \rightarrow m_{i}+c$, with $i=1, \ldots, e$ and $c \in \mathbb{Z}$ : this is the decoupled $\mathrm{U}(1)$ that we have to fix.

Topological factor. The topological $\mathrm{U}(1)_{J}^{e+1}$ fugacities for the naive $\mathrm{U}(2) \times \mathrm{U}(1)^{e}$ theory contribute

$$
z_{0}^{n_{1}+n_{2}} \prod_{i=1}^{e} z_{i}^{m_{i}}
$$

where $z_{0}$ is the fugacity associated to the topological charge of $\mathrm{U}(2)$ and $z_{i}$, with $i=1, \ldots, e$, the fugacity associated to the topological charge of the $i$-th copy of $\mathrm{U}(1)$.

Factoring out the overall U(1). To get rid of the decoupled U(1), which would make this a bad theory, we fix the $\mathbb{Z}$ shift symmetry of the magnetic fluxes and impose a relation on the topological fugacities $z_{I}$, where $I=0,1, \ldots, e$. Different fixings make manifest different topological symmetry enhancements. Here we want to manifest an $\mathrm{SU}(2)^{e}$ enhanced topological symmetry, with one SU(2) per external U(1) node. Therefore we fix the overall $\mathrm{U}(1)$ by imposing

$$
n_{2}=0, \quad z_{0}^{2} \prod_{i=1}^{e} z_{i}=1
$$

In the following we choose to write

$$
z_{0}=\epsilon x_{1} \cdots x_{e}, \quad z_{i}=x_{i}^{-2}, \quad i=1, \ldots, e, \quad \epsilon^{2}=1 .
$$

As we shall see, this choice makes $\mathrm{SU}(2)$ characters manifest in the Hilbert series. $\epsilon$ is the fugacity of a potential discrete $\mathbb{Z}_{2}$ topological symmetry. This $\mathbb{Z}_{2}$ can be absorbed into the center of an $\mathrm{SU}(2)$ symmetry, and correspondingly $\epsilon$ can be absorbed into $z_{i}$ or $x_{i}$, except for the case of no punctures $e=0$, where it is the topological symmetry for the gauge group $\mathrm{SU}(2) / \mathbb{Z}_{2}$. We will sometimes omit $\epsilon$ in the following. 
The monopole formula for Coulomb branch Hilbert series. Following the above discussion, the refined Hilbert series of the Coulomb branch (2.1) reads

$$
\begin{aligned}
H & {[\text { mirror }(g, e)]\left(t ; x_{1}, \ldots, x_{e}\right) } \\
& =\sum_{n_{1} \geq n_{2}=0}^{\infty} \sum_{m_{i} \in \mathbb{Z}} t^{\Delta_{g}\left(n_{1}, n_{2} ; m_{1}, \ldots, m_{e}\right)} P_{\mathrm{U}(1)}(t)^{e}(1-t) P_{\mathrm{U}(2)}\left(t ; n_{1}, n_{2}\right) \epsilon^{n_{1}+n_{2}} \prod_{i=1}^{e} x_{i}^{n_{1}+n_{2}-2 m_{i}},
\end{aligned}
$$

where the last factor comes from (3.2) and (3.4). The classical factors are given by

$$
P_{\mathrm{U}(1)}(t)=\frac{1}{1-t}
$$

and

$$
P_{\mathrm{U}(2)}\left(t ; n_{1}, n_{2}\right)=\left\{\begin{array}{ll}
\frac{1}{(1-t)\left(1-t^{2}\right)}, & n_{1}=n_{2} \\
\frac{1}{(1-t)^{2}}, & n_{1} \neq n_{2}
\end{array} .\right.
$$

The factor $(1-t)$ in front of $P_{\mathrm{U}(2)}$ removes the classical invariants of the decoupled $\mathrm{U}(1)$.

As we show explicitly in subsection 3.1.1, evaluating the monopole formula (3.5) reproduces the refined Hilbert series of the Higgs branch of the mirror theory, formula (7.1) of [11], under the fugacity map $t_{\text {here }}=t_{\text {there }}^{2}$.

Coulomb branch Hilbert series from gluing. It is instructive to rewrite (3.5) as

$$
\begin{aligned}
H[\text { mirror }(g, e)]\left(t ; x_{1}, \ldots, x_{e}\right)= & \sum_{n_{1} \geq n_{2}=0}(1-t) P_{\mathrm{U}(2)}\left(t ; n_{1}, n_{2}\right) t^{(g-1)\left(n_{1}-n_{2}\right)} \times \\
& \epsilon^{n_{1}+n_{2}} \prod_{j=1}^{e} H[T(\mathrm{SU}(2))]\left(t ; x_{j}, x_{j}^{-1} ; n_{1}, n_{2}\right),
\end{aligned}
$$

where $H[T(\mathrm{SU}(2))]$ is the Coulomb branch Hilbert series with background fluxes of the $T(\mathrm{SU}(2))$ theory given by $(2.9)$ with $\boldsymbol{\rho}=(1,1)$ :

$$
\begin{aligned}
H[T(\mathrm{SU}(2))]\left(t ; x, x^{-1} ; n_{1}, n_{2}\right) & =\sum_{m \in \mathbb{Z}} t^{\frac{1}{2}\left(\left|m-n_{1}\right|+\left|m-n_{2}\right|\right)} x^{-2 m} P_{\mathrm{U}(1)}(t) \\
& =t^{\frac{1}{2}\left(n_{1}-n_{2}\right)}(1-t)^{2} \mathrm{PE}\left[\left(1+[2]_{x}\right) t\right] \Psi_{\mathrm{U}(2)}^{\left(n_{1}, n_{2}\right)}\left(x, x^{-1} ; t\right)
\end{aligned}
$$

Eq. (3.8) is nothing but the gluing formula for the Coulomb branch Hilbert series of the star-shaped quiver, which results from gauging the common flavor symmetry of $e$ copies of $T(\mathrm{SU}(2))$ and introducing $g$ adjoint hypermultiplets under the $\mathrm{U}(2)$ group. The gluing factor is

$$
(1-t) P_{\mathrm{U}(2)}\left(t ; n_{1}, n_{2}\right) t^{(g-1)\left|n_{1}-n_{2}\right|} \epsilon^{n_{1}+n_{2}} \prod_{j=1}^{e} x_{j}^{n_{1}+n_{2}},
$$

with $x_{j}^{n_{1}+n_{2}}$ factors already incorporated in $H[T(\mathrm{SU}(2))]$ for convenience. 


\subsubsection{Computation of the Hilbert series for general $g$ and $e$}

We now compute the Coulomb branch Hilbert series (3.8) of the mirror of tri-vertex theories with genus $g$ and $e$ external legs.

Using (2.9), we obtain

$$
\begin{aligned}
H[T(\mathrm{SU}(2))]\left(t ; x, x^{-1} ; n, 0\right) & =t^{\frac{1}{2} n}(1-t)^{2} \mathrm{PE}\left[\left(1+\chi_{[2]}^{\mathrm{SU}(2)}(x)\right) t\right] \Psi_{\mathrm{U}(2)}^{(n, 0)}\left(x, x^{-1} ; t\right) \\
& =t^{\frac{1}{2} n}(1-t) \operatorname{PE}\left[t \chi_{[2]}^{\mathrm{SU}(2)}(x)\right] \Psi_{\mathrm{U}(2)}^{(n, 0)}\left(x, x^{-1} ; t\right),
\end{aligned}
$$

where [2] represents the adjoint representation of $\mathrm{SU}(2)$. An explicit formula for $\Psi_{\mathrm{U}(2)}^{(n, 0)}\left(x, x^{-1} ; t\right)$ is known in terms of $\mathrm{SU}(2)$ characters:

$$
\Psi_{\mathrm{U}(2)}^{(n, 0)}\left(x, x^{-1} ; t\right)=\chi_{[n]}^{\mathrm{SU}(2)}(x)-t \chi_{[n-2]}^{\mathrm{SU}(2)}(x),
$$

where

$$
\chi_{[n]}^{\mathrm{SU}(2)}(x)=\frac{x^{n+1}-x^{-(n+1)}}{x-x^{-1}},
$$

which we extend to $n \in \mathbb{Z}$. Observe that $(1-t) \mathrm{PE}\left[\chi_{[2]}^{\mathrm{SU}(2)}(x) t\right] \Psi_{\mathrm{U}(2)}^{(m, 0)}\left(x, x^{-1} ; t\right)$ is equal to the function $f_{m}(t, x)$ defined in (7.18) of [11]:

$$
\begin{aligned}
f_{m}(t, x) & :=(1-t) \operatorname{PE}\left[\chi_{[2]}^{\mathrm{SU}(2)}(x) t\right] \Psi_{\mathrm{U}(2)}^{(m, 0)}\left(x, x^{-1} ; t\right) \\
& =(1-t)\left(\chi_{[m]}^{\mathrm{SU}(2)}(x)-\chi_{[m-2]}^{\mathrm{SU}(2)}(x) t\right) \mathrm{PE}\left[[2]_{x} t\right] \\
& =\sum_{n=0}^{\infty} \chi_{[2 n+m]}^{\mathrm{SU}(2)}(x) t^{n} .
\end{aligned}
$$

Hence from (3.11) we have

$$
H[T(\mathrm{SU}(2))]\left(t ; x, x^{-1} ; m, 0\right)=t^{\frac{1}{2} m} f_{m}(t, x) .
$$

Substituting this into (3.8), we obtain

$$
\begin{aligned}
& H[\text { mirror }(g, e)]\left(t ; x_{1}, \ldots, x_{e} ; \epsilon\right) \\
& \quad=\sum_{m=0}^{\infty} t^{\frac{1}{2} \chi m} \epsilon^{m}(1-t) P_{\mathrm{U}(2)}(t ; m, 0) \prod_{j=1}^{e} f_{m}\left(t, x_{j}\right) \\
& \quad=\frac{1}{1-t^{2}} \prod_{j=1}^{e} f_{0}\left(t, x_{j}\right)+\sum_{m=1}^{\infty} \frac{t^{\frac{1}{2} \chi m} \epsilon^{m}}{1-t} \prod_{j=1}^{e} f_{m}\left(t, x_{j}\right) \\
& \quad=\frac{1}{1-t^{2}} \sum_{m=0}^{\infty}\left[t^{\frac{1}{2} \chi m} \epsilon^{m} \prod_{j=1}^{e} f_{m}\left(t, x_{j}\right)+t^{\frac{1}{2}(\chi(m+1)+2)} \epsilon^{m+1} \prod_{j=1}^{e} f_{m+1}\left(t, x_{j}\right)\right],
\end{aligned}
$$

where $\chi=2 g+e-2$. This result precisely equals the Higgs branch Hilbert series of the mirror tri-vertex theory, (7.19) of [11], after the redefinition $t \rightarrow t^{2}$ and setting $\epsilon=1$. Note that when $e>0$, the $\mathbb{Z}_{2}$ topological symmetry can be absorbed into the center of any of 


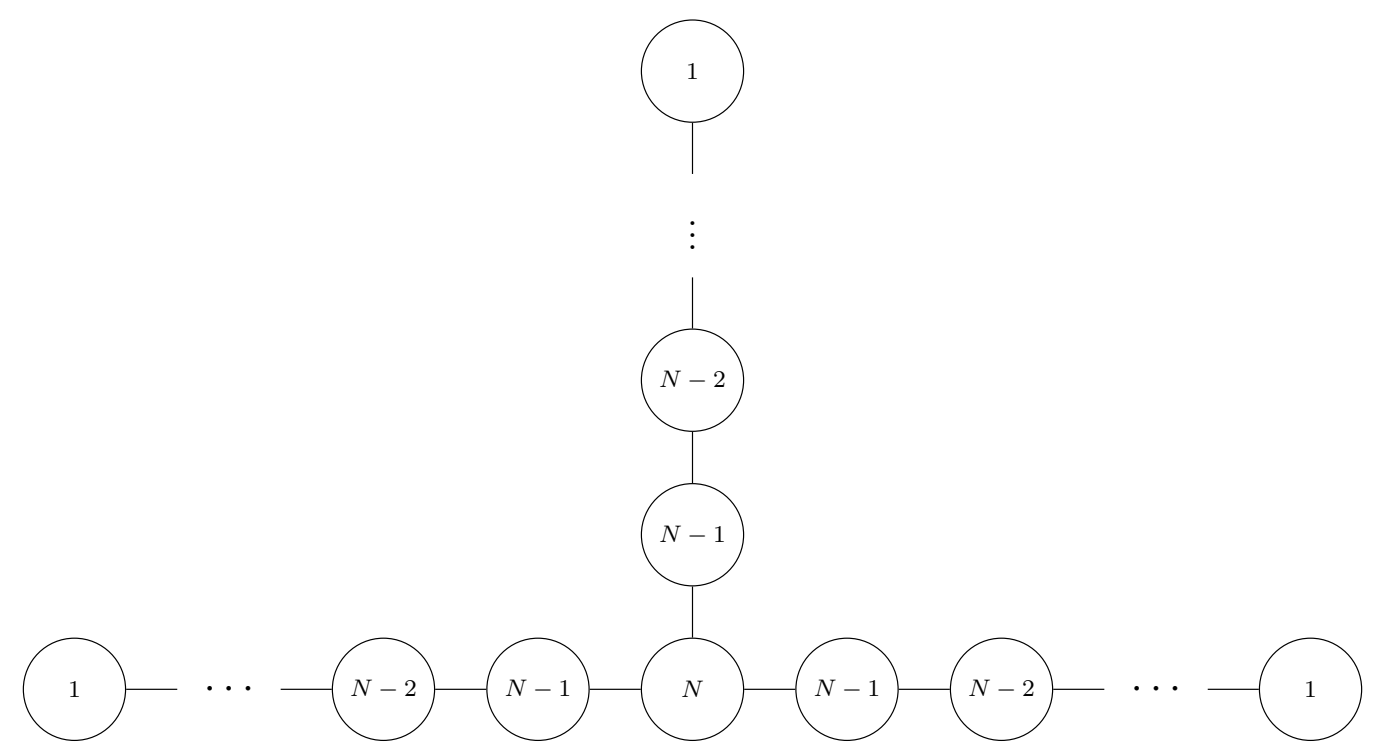

Figure 3. Quiver diagram for the mirror of $T_{N}$. Each node represents a unitary group of the labelled rank and the overall $\mathrm{U}(1)$ is modded out.

the global $\mathrm{SU}(2)$ factors, therefore we can set $\epsilon=1$. When $e=0, \epsilon$ is the fugacity for the actual $\mathbb{Z}_{2}$ topological symmetry of the $\mathrm{SU}(2) / \mathbb{Z}_{2}$ theory with $g$ adjoint hypermultiplets. The Hilbert series of the Coulomb branch is

$$
H[\text { mirror }(g, 0)](t ; \epsilon)=\mathrm{PE}\left[t^{2}+\epsilon\left(t^{g-1}+t^{g}\right)-t^{2 g}\right],
$$

indicating a $\mathbb{C}^{2} / \widehat{D}_{g+1}$ singularity. The monopole generators of dimension $g-1$ and $g$ are odd under $\mathbb{Z}_{2}$. This $\mathbb{Z}_{2}$ symmetry acts on the Higgs branch of the mirror side by flipping sign to any one of the tri-fundamentals in the generators at page 27 of [11].

\subsection{The Coulomb branch of the mirror of $T_{N}$}

The case of a sphere with three maximal punctures $\boldsymbol{\rho}=(1, \cdots, 1)$ is known as the $T_{N}$ theory [5]. We can compute the Coulomb branch Hilbert series of the mirror of the $T_{N}$ theory reduced to three dimensions by gluing three $T(\mathrm{SU}(N))$ tails together. The quiver diagram of such a mirror theory is depicted in figure 3 .

Note that for $N=3$ the quiver of the mirror is the $E_{6}$ quiver and the result should match with the Hilbert series of the reduced moduli space of $1 E_{6}$ instanton on $\mathbb{C}^{2}$.

$$
\begin{aligned}
& H[\text { mirror }\left.T_{N}\right]\left(t ; \boldsymbol{x}^{(1)}, \boldsymbol{x}^{(2)}, \boldsymbol{x}^{(3)}\right) \\
&= \sum_{n_{1} \geq \cdots \geq n_{N}=0}\left\{\prod_{j=1}^{3} H[T(\mathrm{SU}(N))]\left(t ; \boldsymbol{x}^{(j)} ; n_{1}, \ldots, n_{N}\right)\right\} \times \\
& t^{-\delta_{\mathrm{U}(N)}\left(n_{1}, \ldots, n_{N}\right)}(1-t) P_{\mathrm{U}(N)}\left(t ; n_{1}, \ldots, n_{N}\right) \epsilon^{\sum_{i=1}^{N} n_{i}} \\
&= \sum_{n_{1} \geq \cdots \geq n_{N-1} \geq 0} t^{\frac{1}{2} \sum_{j=1}^{N-1}(N+1-2 j) n_{j}}(1-t)^{3 N+1} P_{\mathrm{U}(N)}\left(t ; n_{1}, \ldots, n_{N-1}, 0\right) \times \\
& \epsilon^{\sum_{i=1}^{N-1} n_{i}} \prod_{j=1}^{3} K_{\left(1^{N}\right)}\left(\boldsymbol{x}^{(j)} ; t\right) \Psi_{\mathrm{U}(N)}^{\left(n_{1}, \ldots, n_{N-1}, 0\right)}\left(\boldsymbol{x}^{(j)} ; t\right),
\end{aligned}
$$


where we explain the notation below:

- $\boldsymbol{x}^{(i)}=\left(x_{1}^{(i)}, \ldots, x_{N}^{(i)}\right)$, with $i=1,2,3$, denotes the fugacities of the $\mathrm{SU}(N)$ global symmetry on the Coulomb branch associated with the $i$-th copy of $T(\mathrm{SU}(N))$; they satisfy

$$
\prod_{k=1}^{N} x_{k}^{(i)}=1, \quad \text { with } i=1,2,3 .
$$

- The second line of the first equality is the gluing factor for the $\mathrm{U}(N)$ group:

1. $\delta_{\mathrm{U}(N)}$ denotes the contribution from the $\mathrm{U}(N)$ background vector multiplet:

$$
\begin{aligned}
\delta_{\mathrm{U}(N)}\left(n_{1}, \ldots, n_{N}\right) & =\sum_{1 \leq i<j \leq N}\left|n_{i}-n_{j}\right| \\
& =\sum_{j=1}^{N}(N+1-2 j) n_{j}, \quad n_{1} \geq n_{2} \geq \cdots \geq n_{N} \geq 0 .
\end{aligned}
$$

2. The removal of the overall $\mathrm{U}(1)$ is done in two steps:

(a) Multiplying $(1-t)$ to the function $P_{\mathrm{U}(N)}\left(t ; n_{1}, \ldots, n_{N}\right)$.

(b) Restricting $n_{N}=0$.

- The prefactor $K_{\left(1^{N}\right)}(\boldsymbol{x} ; t)$ is given by

$$
K_{\left(1^{N}\right)}(\boldsymbol{x} ; t)=\operatorname{PE}\left[\chi_{\mathbf{A d j}}^{\mathrm{U}(N)}(\boldsymbol{x}) t\right] .
$$

- The fugacity $\epsilon$, with $\epsilon^{N}=1$, corresponds to a potential $\mathbb{Z}_{N}$ discrete topological symmetry for the $\mathrm{U}(N)$ gauge group modulo $\mathrm{U}(1)$. In the notations of section 3 of [1], the $\mathbb{Z}_{N}$ valued fugacity is related to the ambiguity in taking the $N$-th root when solving the constraint on the topological fugacities for $z_{0}$ :

$$
z_{0}=\epsilon \widehat{z}_{0}, \quad \text { with } \quad \widehat{z}_{0}:=\left(\prod_{a=1}^{e} \prod_{k=1}^{d_{a}} z_{k, a}^{N_{k, a}}\right)^{1 / N}
$$

where $\widehat{z}_{0}$ denotes the $N$-th principal root and $\epsilon$ runs over $N$-th roots of unity,

$$
\epsilon^{N}=1
$$

and $N_{k, a}$ and $z_{k, a}$ are the rank and the fugacity for the topological symmetry of the $k$-th gauge group in the $a$-th leg. Often all or part of this $\mathbb{Z}_{N}$ symmetry can be absorbed in the center of the continuous topological symmetry associated to $z_{k, a}$. For this reason we will sometimes omit $\epsilon$ in the following. 
Our result should agree with the Higgs branch Hilbert series of the $T_{N}$ theory. The latter can be evaluated in the $4 \mathrm{~d}$ version of the theory, since the Higgs branch does not depend on the dimension. Let us compare (3.18) with the result in [4] for the Higgs branch Hilbert series of $T_{N}$ which is computed by the $4 \mathrm{~d}$ Hall-Littlewood index. In that reference, the HL polynomial is defined with a normalization factor: ${ }^{4}$

$$
\widehat{\Psi}_{\mathrm{U}(N)}^{\lambda}\left(x_{1}, \ldots, x_{N} ; t\right)=\mathcal{N}_{\boldsymbol{\lambda}}(t) \Psi_{\mathrm{U}(N)}^{\lambda}\left(x_{1}, \ldots, x_{N} ; t\right) .
$$

The normalization $\mathcal{N}_{\boldsymbol{\lambda}}(t)$ is given by

$$
\mathcal{N}_{\lambda_{1}, \ldots \lambda_{k}}^{-2}(t)=\prod_{i=0}^{\infty} \prod_{j=1}^{m(i)}\left(\frac{1-t^{j}}{1-t}\right)
$$

where $m(i)$ is the number of rows in the Young diagram $\boldsymbol{\lambda}=\left(\lambda_{1}, \ldots, \lambda_{N}\right)$ of length $i$. It is related to $P_{\mathrm{U}(N)}$ as follows:

$$
(1-t)^{N} P_{\mathrm{U}(N)}\left(t ; n_{1}, \ldots, n_{N-1}, 0\right)=\mathcal{N}_{n_{1}, \ldots, n_{N-1}, 0}(t)^{2} .
$$

Using the identity

$$
(1-t)^{2 N+1} t^{\frac{1}{2} \sum_{j=1}^{N-1}(N+1-2 j) n_{j}}=\frac{(1-t)^{N+2} \prod_{i=2}^{N}\left(1-t^{i}\right)}{\Psi_{\mathrm{U}(N)}^{\left(n_{1}, \ldots, n_{N-1}, 0\right)}\left(t^{\frac{1}{2}(N-1)}, t^{\frac{1}{2}(N-3)}, \ldots, t^{-\frac{1}{2}(N-1)} ; t\right)},
$$

we arrive at

$$
\begin{aligned}
& H\left[\text { mirror } T_{N}\right]\left(t ; \boldsymbol{x}^{(1)}, \boldsymbol{x}^{(2)}, \boldsymbol{x}^{(3)}\right) \\
& =(1-t)^{N+2}\left\{\prod_{i=2}^{N}\left(1-t^{i}\right)\right\} K_{\left(1^{N}\right)}\left(\boldsymbol{x}^{(1)} ; t\right) K_{\left(1^{N}\right)}\left(\boldsymbol{x}^{(2)} ; t\right) K_{\left(1^{N}\right)}\left(\boldsymbol{x}^{(3)} ; t\right) \times \\
& \quad \sum_{n_{1} \geq n_{2} \geq \cdots \geq n_{N-1} \geq 0} \frac{\widehat{\Psi}_{\mathrm{U}(N)}^{\left(n_{1}, \ldots, n_{N-1}, 0\right)}\left(\boldsymbol{x}^{(1)} ; t\right) \widehat{\Psi}_{\mathrm{U}(N)}^{\left(n_{1}, \ldots, n_{N-1}, 0\right)}\left(\boldsymbol{x}^{(2)} ; t\right) \widehat{\Psi}_{\mathrm{U}(N)}^{\left(n_{1}, \ldots, n_{N-1}, 0\right)}\left(\boldsymbol{x}^{(3)} ; t\right)}{\widehat{\Psi}_{\mathrm{U}(N)}^{\left(n_{1}, \ldots ., n_{N-1}, 0\right)}\left(t^{\frac{1}{2}(N-1)}, t^{\frac{1}{2}(N-3)}, \ldots, t^{-\frac{1}{2}(N-1)} ; t\right)},
\end{aligned}
$$

where the normalized HL polynomial $\widehat{\Psi}_{\mathrm{U}(N)}^{\boldsymbol{n}}(\boldsymbol{x} ; t)$ is defined as in (3.24). Our result agrees with formula (5.33) of [4].

\subsection{The Coulomb branch of the mirror of a general $3 d$ Sicilian theory}

The computation of the Coulomb branch Hilbert series for the mirror of $T_{N}$ can be easily generalized to a general $3 d$ Sicilian theory. For the mirror of a theory that arises from a compactification of the $A_{N-1} 6 d(2,0)$ theory on a circle times a genus $g$ Riemann surface with punctures $\left\{\boldsymbol{\rho}_{1}, \boldsymbol{\rho}_{2}, \ldots, \boldsymbol{\rho}_{e}\right\}$, the Coulomb branch Hilbert series is given by

$$
\begin{aligned}
& H\left[\text { mirror } g,\left\{\boldsymbol{\rho}_{1}, \boldsymbol{\rho}_{2}, \ldots, \boldsymbol{\rho}_{e}\right\}\right]\left(t ; \boldsymbol{x}^{(1)}, \ldots, \boldsymbol{x}^{(e)}\right) \\
& =\sum_{\substack{n_{1} \geq \cdots \geq n_{N}=0 \\
\widetilde{\delta}_{\mathrm{U}(N), g}\left(n_{1}, \ldots, n_{N}\right)}}\left\{\prod_{j=1}^{e} H\left[T_{\boldsymbol{\rho}_{j}}(\mathrm{SU}(N))\right]\left(t ; \boldsymbol{x}^{(j)} ; n_{1}, \ldots, n_{N}\right)\right\} \times \\
& t_{\mathrm{U}(N)}\left(t ; n_{1}, \ldots, n_{N}\right),
\end{aligned}
$$

\footnotetext{
${ }^{4}$ Our fugacity $t$ is related to $\tau$ in [4] by $\tau=t^{1 / 2}$.
} 
where the contribution of the $g \mathrm{U}(N)$ adjoint hypermultiplets and vector multiplet to the dimension of monopole operators is

$$
\begin{aligned}
\widetilde{\delta}_{\mathrm{U}(N), g}(\boldsymbol{n}) & =(g-1) \delta_{\mathrm{U}(N)}(\boldsymbol{n})=(g-1) \sum_{1 \leq i<j \leq N}\left|n_{i}-n_{j}\right| \\
& =(g-1) \sum_{j=1}^{N}(N+1-2 j) n_{j}, \quad n_{1} \geq \cdots \geq n_{N} \geq 0,
\end{aligned}
$$

with $\delta_{\mathrm{U}(N)}\left(n_{1}, \ldots, n_{N}\right)$ given by $(3.30)$. We therefore obtain

$$
\begin{aligned}
& H\left[\text { mirror } g,\left\{\boldsymbol{\rho}_{1}, \boldsymbol{\rho}_{2}, \ldots, \boldsymbol{\rho}_{e}\right\}\right]\left(t ; \boldsymbol{x}^{(1)}, \ldots, \boldsymbol{x}^{(e)}\right) \\
& \quad=\sum_{n_{1} \geq \cdots \geq n_{N-1} \geq 0} t^{\left(\frac{e}{2}+g-1\right) \sum_{j=1}^{N-1}(N+1-2 j) n_{j}}(1-t)^{e N+1} P_{\mathrm{U}(N)}\left(t ; n_{1}, \ldots, n_{N-1}, 0\right) \times \\
& \quad \prod_{j=1}^{e} K_{\boldsymbol{\rho}_{j}}\left(\boldsymbol{x}^{(j)} ; t\right) \Psi_{\mathrm{U}(N)}^{\left(n_{1}, \ldots, n_{N-1}, 0\right)}\left(\boldsymbol{x}^{(j)} t^{\frac{1}{2} \boldsymbol{w}_{\rho_{j}}} ; t\right),
\end{aligned}
$$

\subsubsection{The case of genus zero}

In a special case of $g=0$, we use (3.26) and (3.27) to obtain

$$
\begin{aligned}
& H\left[\text { mirror }\left\{\boldsymbol{\rho}_{1}, \boldsymbol{\rho}_{2}, \ldots, \boldsymbol{\rho}_{e}\right\}\right]\left(t ; \boldsymbol{x}^{(1)}, \ldots, \boldsymbol{x}^{(e)}\right) \\
& =(1-t)^{e+(N-1)}\left\{\prod_{i=2}^{N}\left(1-t^{i}\right)\right\}^{e-2} \times \\
& \quad \sum_{n_{1} \geq n_{2} \geq \cdots \geq n_{N-1} \geq 0} \frac{\prod_{j=1}^{e} K_{\boldsymbol{\rho}_{j}}\left(\boldsymbol{x}^{(j)} ; t\right) \widehat{\Psi}_{\mathrm{U}(N)}^{\left(n_{1}, \ldots, n_{N-1}, 0\right)}\left(\boldsymbol{x}^{(j)} t^{\frac{1}{2} \boldsymbol{w}_{\rho_{j}}} ; t\right)}{\left[\widehat{\Psi}_{\mathrm{U}(N)}^{\left(n_{1}, \ldots, n_{N-1}, 0\right)}\left(t^{\frac{1}{2}(N-1)}, t^{\frac{1}{2}(N-3)}, \ldots, t^{-\frac{1}{2}(N-1)} ; t\right)\right]^{e-2}},
\end{aligned}
$$

where $\widehat{\Psi}_{\mathrm{U}(N)}$ denotes the normalized Hall-Littlewood polynomial defined in (3.24). This result agrees with the Higgs branch Hilbert series of the Gaiotto theory, computed as a Hall-Littlewood index for $g=0$ in (2.13) of [9].

As discussed in [9], the formula (3.31) can be used to write the Hilbert series for the moduli spaces of $E_{6}, E_{7}$ and $E_{8}$ instantons on $\mathbb{C}^{2}$, which can be realized as the Higgs branch of the $6 d(2,0)$ theory compactified on a Riemann sphere with punctures $\left\{\boldsymbol{\rho}_{1}, \boldsymbol{\rho}_{2}, \boldsymbol{\rho}_{3}\right\}$

$$
\begin{array}{llll} 
& \multicolumn{1}{c}{\boldsymbol{\rho}_{1}} & \multicolumn{1}{c}{\boldsymbol{\rho}_{2}} & \multicolumn{1}{c}{\boldsymbol{\rho}_{3}} \\
E_{6} & (k, k, k) & (k, k, k) & (k, k, k-1,1) \\
E_{7} & (k, k, k, k) & (2 k, 2 k) & (k, k, k, k-1,1) \\
E_{8} & (3 k, 3 k) & (2 k, 2 k, 2 k) & (k, k, k, k, k, k-1,1)
\end{array}
$$

corresponding to the mirror quiver given in figure 4 .

\subsubsection{Mirror of the SU(3) Sicilian theory with $g=1$ and a maximal puncture}

Recall that for genus $g>0$ the HL index differs from the Higgs branch Hilbert series of the Sicilian theory [4]. The latter is given by our formula (3.30), assuming mirror symmetry. 

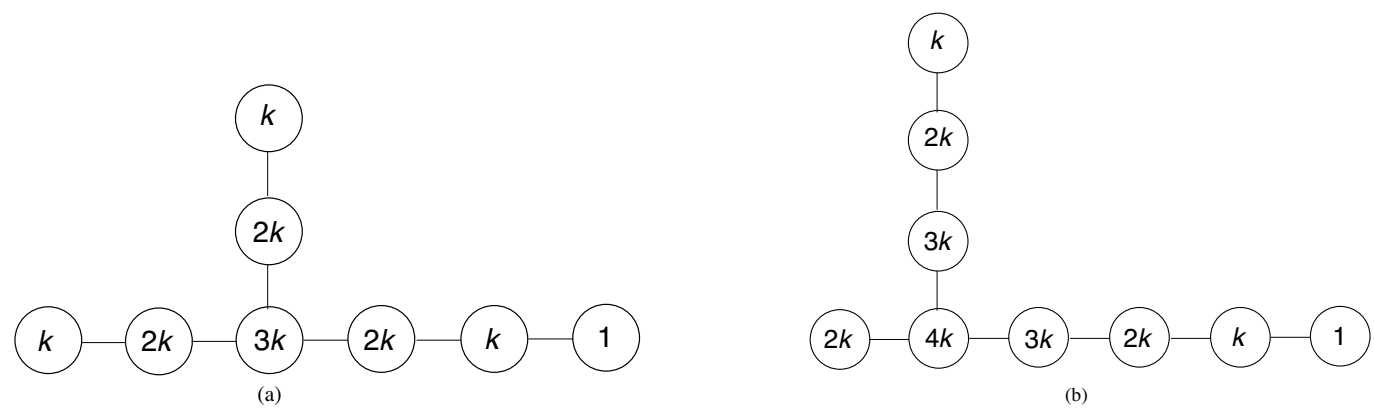

(b)

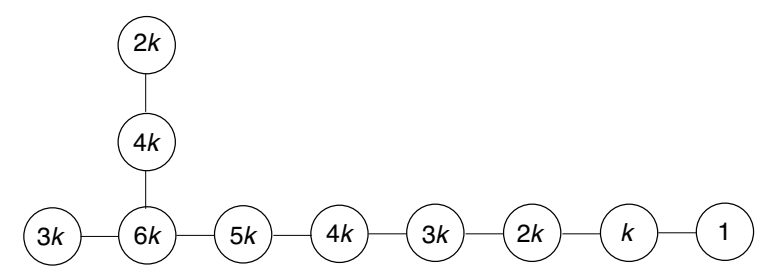

(c)

Figure 4. The moduli spaces of $k E_{6}, E_{7}$ and $E_{8}$ instantons on $\mathbb{C}^{2}$ can be realized using the Coulomb branch of quiver diagrams (a), (b) and (c) respectively. Each node represents a unitary group of the labelled rank and the overall $\mathrm{U}(1)$ is modded out in each diagram.

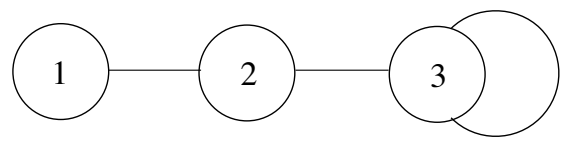

Figure 5. Quiver for the mirror of the $A_{2}$ theory on a circle times a torus with one maximal puncture. The overall $\mathrm{U}(1)$ is factored out.

Let us provide an explicit example for the case of $N=3, g=1$ and one maximal puncture $\boldsymbol{\rho}=(1,1,1)$ below. The quiver diagram of the mirror theory of our interest is depicted in figure 5 . This example is particularly interesting because the global symmetry on the Coulomb branch enhances to $G_{2}$ [9]. We will show this by computing the Hilbert series and expanding it in $G_{2}$ characters.

The Coulomb branch Hilbert series can be computed using (3.30), where the fugacities $x_{1}, x_{2}, x_{3}$ are related to the fugacities for the topological charges of $\mathrm{U}(1), \mathrm{U}(2)$ and $\mathrm{U}(3)$ gauge groups and are subject to the constraint (2.13). In order to make $G_{2}$ characters manifest in the Hilbert series, we use the fugacity map ${ }^{5}$

$$
x_{1}=y_{1}, \quad x_{2}=y_{1} y_{2}^{-1}, \quad x_{3}=y_{1}^{-2} y_{2},
$$

where $x_{1}, x_{2}$ are the fugacities in formula (3.30) and $y_{1}, y_{2}$ are the $G_{2}$ fugacities.

We then obtain

$$
H[\text { mirror } g=1,(1,1,1)]\left(t ; y_{1}, y_{1} y_{2}^{-1}, y_{2} y_{1}^{-2}\right)=f(0,0,0)+f(3,1,5),
$$

\footnotetext{
${ }^{5}$ Here we use the characters of $G_{2}$ as in LiE online service at the following link: http://young.sp2mi.univpoitiers.fr/cgi-bin/form-prep/marc/LiE_form.act?action=character\&type=G\&rank=2\&highest_rank=8.
} 
where

$$
f(a, b, c)=\sum_{n_{1}=0}^{\infty} \sum_{n_{2}=0}^{\infty} \sum_{n_{3}=0}^{\infty} \sum_{n_{4}=0}^{\infty}\left[2 n_{2}+3 n_{3}+a, n_{1}+2 n_{4}+b\right] t^{n_{1}+2 n_{2}+3 n_{3}+4 n_{4}+c},
$$

and $[a, b]$ denotes the character of the $G_{2}$ representation with highest weight $[a, b]$, written in terms of $y_{1}, y_{2}$. The character expansion (3.34) shows not only that the adjoint representation arises at $\Delta=1$ (for the scalar partners of conserved currents), but also that the whole chiral spectrum transforms in $G_{2}$ representations as expected.

The unrefined Hilbert series is given by

$$
H[\text { mirror } g=1,(1,1,1)](t ; 1,1,1)=\frac{1+4 t+9 t^{2}+9 t^{3}+4 t^{4}+t^{5}}{(1-t)^{10}},
$$

with a palindromic numerator and a pole at $t=1$ of order 10 , equal to the complex dimension of the Coulomb branch of the moduli space.

The generating function of highest weights [24]. The highest weight vectors that appear in formula (3.34) can be collected in the following generating function:

$$
\begin{aligned}
& \mathrm{PE}\left[\mu_{2} t+\mu_{1}^{2} t^{2}+\mu_{1}^{3} t^{3}+\mu_{2}^{2} t^{4}+\mu_{1}^{3} \mu_{2} t^{5}-\mu_{1}^{6} \mu_{2}^{2} t^{10}\right] \\
& \quad=\frac{1-t^{10} \mu_{1}^{6} \mu_{2}^{2}}{\left(1-t^{2} \mu_{1}^{2}\right)\left(1-t^{3} \mu_{1}^{3}\right)\left(1-t \mu_{2}\right)\left(1-t^{5} \mu_{1}^{3} \mu_{2}\right)\left(1-t^{4} \mu_{2}^{2}\right)},
\end{aligned}
$$

where $\mu_{1}$ and $\mu_{2}$ are the fugacities associated with the highest weights $n_{1}$ and $n_{2}$ of representations of $G_{2}$. Upon computing the power series in $t$ of (3.37), the powers $\mu_{1}^{n_{1}} \mu_{2}^{n_{2}}$ can be traded for the Dynkin label $\left[n_{1}, n_{2}\right]$ to obtain the character expansion as stated in (3.34). Let us demonstrate this for the first few terms in the power series:

$$
1+\mu_{2} t+\left(\mu_{1}^{2}+\mu_{2}^{2}\right) t^{2}+\left(\mu_{1}^{3}+\mu_{1}^{2} \mu_{2}+\mu_{2}^{3}\right) t^{3}+\ldots .
$$

Trading the powers of $\mu_{1}$ and $\mu_{2}$ for the Dynkin label, we obtain

$$
1+[0,1] t+([2,0]+[0,2]) t^{2}+([3,0]+[2,1]+[0,3]) t^{3}+\ldots .
$$

\section{Mirrors of $3 d$ Sicilian theories of $D$-type}

In this section we consider three dimensional theories arising from the $6 d(2,0)$ theory of $D_{N}$ type compactified on a circle times a Riemann surface with punctures. Each puncture is classified by a $D$-partition of $\mathrm{SO}(2 N)$. The Coulomb branch Hilbert series of the mirror theory can be computed by gluing copies of the $T_{\boldsymbol{\rho}}(\mathrm{SO}(2 N))$ theories [6] according to the general discussion in section 3 . The quivers for the $T_{\boldsymbol{\rho}}(\mathrm{SO}(2 N))$ theories, which can be realised from brane and orientifold configurations as in [25], are reviewed in section 4.2 of [3]. We remark that we gauge the centerless group $\mathrm{SO}(2 N) / \mathbb{Z}_{2}$ rather than $\mathrm{SO}(2 N)$. Consequently, the magnetic fluxes of the gluing gauge group belong to the weight lattice of the dual group $\operatorname{Spin}(2 N)$ modulo the Weyl group. 
Given a $3 d$ Sicilian theory with genus $g$ and $e D$-type punctures $\left\{\boldsymbol{\rho}_{1}, \boldsymbol{\rho}_{2}, \ldots, \boldsymbol{\rho}_{e}\right\}$, the Coulomb branch Hilbert series of its mirror theory is ${ }^{6}$

$$
\begin{aligned}
& H\left[\text { mirror } g,\left\{\boldsymbol{\rho}_{1}, \boldsymbol{\rho}_{2}, \ldots, \boldsymbol{\rho}_{e}\right\}\right]\left(t ; \boldsymbol{x}^{(1)}, \ldots, \boldsymbol{x}^{(e)}\right) \\
& =\sum_{\substack{n_{1} \geq \cdots \geq n_{N-1} \geq\left|n_{N}\right| \\
\widetilde{\delta}_{\mathrm{SO}(2 N), g}\left(n_{1}, \ldots, n_{N}\right)}}\left\{\prod_{\substack{\mathrm{SO}(2 N) \\
t}}^{e}\left(t ; n_{1}, \ldots, n_{\boldsymbol{\rho}_{j}}(\mathrm{SO}(2 N))\right]\left(t ; \boldsymbol{x}^{(j)} ; n_{1}, \ldots, n_{N}\right)\right\} \times
\end{aligned}
$$

where $H\left[T_{\boldsymbol{\rho}}(\mathrm{SO}(2 N))\right]$ is given by (2.21), the Casimir factor $P_{\mathrm{SO}(2 N)}$ is computed as in (2.2) (see (A.10) of [1] for an explicit expression), and $\widetilde{\delta}_{\mathrm{SO}(2 N), g}(\boldsymbol{n})$ is the contribution of the $g \mathrm{SO}(2 N)$ adjoint hypermultiplets and vector multiplet to the dimension of monopole operators is

$$
\widetilde{\delta}_{\mathrm{SO}(2 N), g}(\boldsymbol{n})=(g-1) \delta_{\mathrm{SO}(2 N)}(\boldsymbol{n})=(g-1) \sum_{j=1}^{N-1}(2 N-2 j) n_{j},
$$

with the second equality following from (2.24). Note that because the dual of the gluing group is $\operatorname{Spin}(2 N), n_{1}, \ldots, n_{N}$ are all integers or all half-odd integers.

For $g=0$ our formula (4.1) for the Coulomb branch Hilbert series of mirrors of Dtype Sicilian theories proposed in [6] agrees with the Higgs branch Hilbert series of the Sicilian theory, computed as the Hall-Littlewood limit of the superconformal index of the $4 \mathrm{~d}$ Sicilian theory in formula (4.10) of $[10]{ }^{7}$ For higher genus the HL index does not compute the Hilbert series of the Higgs branch. Formula (4.1) provides a prediction for the latter, assuming mirror symmetry.

In the rest of the section we provide examples of Sicilian theories with $D_{3}$ and $D_{4}$ symmetry and we compare with the results in $[10,26]$. We start this section by considering the case of $D_{3}$. Due to the isomorphism of its Lie algebra with that of $A_{3}$, each $D_{3}$ puncture can be identified with an $A_{3}$ puncture. We compute the Coulomb branch Hilbert series of mirror theories of $3 d$ Sicilian theories with $D_{3}$ punctures using the Hall-Littlewood formula and compare the result with those with $A_{3}$ punctures. We then consider $D_{4}$ theories with a set of punctures for which the Higgs branch is explicitly known and we compare our result for the Coulomb branch Hilbert series of the mirror with the Higgs branch Hilbert series. The case of twisted D punctures is discussed in the appendix. All these examples demonstrate the validity of our formula (4.1).

\section{1 $D_{3}$ punctures}

There are four possible $D$-partitions of $\mathrm{SO}(6)$. These partitions and the identification with $A_{3}$ partitions are given on page 17 of [26]. We list them as follows in table 1 . Next, we

\footnotetext{
${ }^{6}$ It is straightforward to include in (4.1) a fugacity for the center of $\operatorname{Spin}(2 N)$, but we prefer not to clutter formulae with those factors, which can often be reabsorbed.

${ }^{7}$ The orthonormal Hall-Littlewood polynomials used in [10] can be expressed in terms of the HallLittlewood polynomials used here as $P_{M}^{n}(\boldsymbol{a} \mid 0, t)=(1-t)^{r k(G) / 2} P_{G^{\vee}}(t ; \boldsymbol{n})^{1 / 2} \Psi_{G}^{n}(\boldsymbol{a}(t, \boldsymbol{x}) ; t)$. The pre-factors are related by $\mathcal{K}_{G}=(1-t)^{r k(G) / 2} K^{G}$. Finally, for $G=\operatorname{SO}(2 N)$ one finds $\mathcal{A}(0, t) / P_{M \operatorname{SO}(2 N)}^{n}\left(1, t, t^{2}, \ldots, t^{N-1} \mid 0, t\right)$ $=t^{\frac{1}{2} \delta_{\mathrm{SO}(2 N)}{ }^{(\boldsymbol{n})}} P_{\mathrm{SO}(2 N)}(t ; \boldsymbol{n})^{-1 / 2}$.
} 


\begin{tabular}{|c|c|c|}
\hline$D_{3}$ puncture & $A_{3}$ puncture & Global symmetry \\
\hline$\left(1^{6}\right)$ & $\left(1^{4}\right)$ & $\mathrm{SO}(6) \simeq \mathrm{SU}(4)$ \\
\hline$\left(2^{2}, 1^{2}\right)$ & $\left(2,1^{2}\right)$ & $\mathrm{USp}(2) \times \mathrm{SO}(2) \simeq \mathrm{SU}(2) \times \mathrm{U}(1)$ \\
\hline$\left(3,1^{3}\right)$ & $\left(2^{2}\right)$ & $\mathrm{SO}(3) \simeq \mathrm{SU}(2)$ \\
\hline$\left(3^{2}\right)$ & $(3,1)$ & $\mathrm{SO}(2) \simeq \mathrm{U}(1)$ \\
\hline
\end{tabular}

Table 1. The list of $D_{3}$ regular punctures, their identifications with $A_{3}$ punctures and the associated global symmetries.

consider an example of the mirror theory of a $3 d$ Sicilian theory with $D_{3}$ punctures $\left(3^{2}\right)$, $\left(1^{6}\right)$ and $\left(1^{6}\right)$.

\subsection{1 $D_{3}$ punctures: $\left(3^{2}\right),\left(1^{6}\right)$ and $\left(1^{6}\right)$}

In terms of $A_{3}$ punctures, these punctures correspond to two maximal ( $\left.1^{4}\right)$ and one minimal $(3,1)$ punctures. This Sicilian theory corresponds to the quiver diagram $[\mathrm{SU}(4)]-[\mathrm{SU}(4)]$, and contains 16 free hypermultiplets; see [5] and page 18 of [27].

The Coulomb branch Hilbert series of the mirror theory of this Sicilian theory can be computed by gluing two copies of $T_{\left(1^{6}\right)}(\mathrm{SO}(6))$ and one copy of $T_{\left(3^{2}\right)}(\mathrm{SO}(6))$ together via the common $\mathrm{SO}(6)$ symmetry:

$$
\begin{aligned}
H(t ; \boldsymbol{x}, \boldsymbol{y}, z)= & \sum_{a_{1}, a_{2}, a_{3} \geq 0} t^{-\delta_{\mathrm{SO}(6)}(\boldsymbol{n}(\boldsymbol{a}))} P_{\mathrm{SO}(6)}(t ; \boldsymbol{n}(\boldsymbol{a})) H\left[T_{\left(1^{6}\right)}(\mathrm{SO}(6))\right](t ; \boldsymbol{x} ; \boldsymbol{n}(\boldsymbol{a})) \times \\
& H\left[T_{\left(1^{6}\right)}(\mathrm{SO}(6))\right](t ; \boldsymbol{y} ; \boldsymbol{n}(\boldsymbol{a})) H\left[T_{\left(3^{2}\right)}(\mathrm{SO}(6))\right](t ; z ; \boldsymbol{n}(\boldsymbol{a})),
\end{aligned}
$$

where $\boldsymbol{x}, \boldsymbol{y}, z$ are respectively fugacities of $\mathrm{SO}(6), \mathrm{SO}(6)$ and $\mathrm{SO}(2)$ symmetries and the function $P_{\mathrm{SO}(6)}$ is defined as in (A.10) of [1], and

$$
\begin{aligned}
\boldsymbol{n}(\boldsymbol{a}) & =\left(a_{1}+\frac{1}{2}\left(a_{2}+a_{3}\right), \frac{1}{2}\left(a_{2}+a_{3}\right), \frac{1}{2}\left(-a_{2}+a_{3}\right)\right), \\
\delta_{\mathrm{SO}(6)}(\boldsymbol{n}) & =4 n_{1}+2 n_{2}, \\
H\left[T_{\left(1^{6}\right)}(\mathrm{SO}(6))\right](t ; \boldsymbol{x} ; \boldsymbol{n}) & =t^{\frac{1}{2} \delta_{\mathrm{SO}(6)}(\boldsymbol{n})}(1-t)^{3} \mathrm{PE}\left[t \chi_{[0,1,1]}^{D_{3}}(\boldsymbol{x})\right] \Psi_{D_{3}}(\boldsymbol{x} ; \boldsymbol{n} ; t), \\
H\left[T_{\left(3^{2}\right)}(\mathrm{SO}(6))\right](t ; x ; \boldsymbol{n}) & =t^{\frac{1}{2} \delta_{\mathrm{SO}(6)}(\boldsymbol{n})}(1-t)^{3} \mathrm{PE}\left[t+t^{2} \chi_{[2]}^{C_{1}}(x)+t^{3}\right] \Psi_{D_{3}}\left(t x, t^{-1} x, x ; \boldsymbol{n} ; t\right) .
\end{aligned}
$$

Note that in the above notation, $\boldsymbol{a}=\left[a_{1}, a_{2}, a_{3}\right]$ denotes a Dynkin label of an irreducible representation of $\operatorname{Spin}(6)$ and $\boldsymbol{n}=\left(n_{1}, n_{2}, n_{3}\right)$ denotes its highest weight in the standard basis. Hence the summations run over all irreducible representations of Spin(6), including the spinorial representations.

It can be checked that the first few terms in the power series of (4.3) are equal to those of the Hilbert series of 16 free hypermultiplets in the spinor representations of $\mathrm{SO}(6)$, as 


\begin{tabular}{|l|l|}
\hline Partition $\boldsymbol{\rho}$ & Quiver diagram for $T_{\boldsymbol{\rho}}(\mathrm{SO}(8))$ \\
\hline$(5,3)$ & {$[\mathrm{SO}(8)]-(\mathrm{USp}(2))$} \\
$\left(3^{2}, 1^{2}\right)$ & {$[\mathrm{SO}(8)]-(\mathrm{USp}(4))-(\mathrm{SO}(2))$} \\
$\left(2^{4}\right)$ & {$[\mathrm{SO}(8)]-(\mathrm{USp}(6))-(\mathrm{SO}(4))-(\mathrm{USp}(2))$} \\
$\left(3,1^{5}\right)$ & {$[\mathrm{SO}(8)]-(\mathrm{USp}(4))-(\mathrm{SO}(4))-(\mathrm{USp}(2))-(\mathrm{SO}(2))$} \\
$\left(2^{2}, 1^{4}\right)$ & {$[\mathrm{SO}(8)]-(\mathrm{USp}(6))-(\mathrm{SO}(4))-(\mathrm{USp}(2))-(\mathrm{SO}(2))$} \\
$\left(1^{8}\right)$ & {$[\mathrm{SO}(8)]-(\mathrm{USp}(6))-(\mathrm{SO}(6))-(\mathrm{USp}(4))-(\mathrm{SO}(4))-(\mathrm{USp}(2))-(\mathrm{SO}(2))$} \\
\hline
\end{tabular}

Table 2. Quiver diagrams for $T_{\boldsymbol{\rho}}(\mathrm{SO}(8))$ for certain $D_{4}$ partitions $\boldsymbol{\rho}$.

expected from mirror symmetry:

$$
\begin{aligned}
H(t ; \boldsymbol{x}, \boldsymbol{y}, z)= & \operatorname{PE}\left[\left\{z^{1 / 2} \chi_{[0,1,0]}^{D_{3}}(\boldsymbol{x}) \chi_{[0,1,0]}^{D_{3}}(\boldsymbol{y})+z^{-1 / 2} \chi_{[0,0,1]}^{D_{3}}(\boldsymbol{x}) \chi_{[0,0,1]}^{D_{3}}(\boldsymbol{y})\right\} t\right] \\
= & \sum_{n_{1}, n_{2}, n_{3}=0}^{\infty} \chi_{\left[n_{2}, n_{1}, n_{3}\right]}^{D_{3}}(\boldsymbol{x}) \chi_{\left[n_{2}, n_{1}, n_{3}\right]}^{D_{3}}(\boldsymbol{y})\left(z^{1 / 2} t\right)^{n_{1}+2 n_{2}+3 n_{3}} \times \\
& \sum_{m_{1}, m_{2}, m_{3}=0}^{\infty} \chi_{\left[m_{2}, m_{3}, m_{1}\right]}^{D_{3}}(\boldsymbol{x}) \chi_{\left[m_{2}, m_{3}, m_{1}\right]}^{D_{3}}(\boldsymbol{y})\left(z^{-1 / 2} t\right)^{m_{1}+2 m_{2}+3 m_{3}}
\end{aligned}
$$

\section{$4.2 \quad D_{4}$ punctures}

In this section, we provide three examples on Sicilian theories with the following $D_{4}$ punctures.

1. $(5,3),\left(2^{2}, 1^{4}\right)$ and $\left(1^{8}\right)$,

2. $\left(3^{2}, 1^{2}\right),\left(2^{2}, 1^{4}\right)$ and $\left(2^{2}, 1^{4}\right)$,

3. $(5,3),(5,3),\left(2^{4}\right)$ and $\left(3,1^{5}\right)$.

In the following subsections, we compute the Coulomb branch Hilbert series of the mirror theories of these Sicilian theories and compare the results to those presented in [26].

For reference, we tabulate the quiver diagrams for $T_{\boldsymbol{\rho}}(\mathrm{SO}(8)$, with $\boldsymbol{\rho}$ being partitions listed above, in table 2.

\subsection{1 $D_{4}$ punctures: $(5,3),\left(2^{2}, 1^{4}\right)$ and $\left(1^{8}\right)$}

The global symmetries associated with these punctures are trivial, $\operatorname{USp}(2) \times \mathrm{SO}(4) \simeq$ $\mathrm{SU}(2)^{3}$ and $\mathrm{SO}(8)$, respectively. According to page 24 of [26], this Sicilian theory is a free theory containing 48 half-hypermultiplets.

We realize the Higgs branch of this theory from the Coulomb branch of the mirror theory. The quiver diagram of the latter can be obtained by gluing the quiver diagrams of $T_{(5,3)}(\mathrm{SO}(8)), T_{\left(2^{2}, 1^{4}\right)}(\mathrm{SO}(8))$ and $T_{\left(1^{8}\right)}(\mathrm{SO}(8))$ via the common symmetry $\mathrm{SO}(8) / \mathbb{Z}_{2}$; this is depicted in (4.6), where each gray node labeled by $N$ denotes an $\mathrm{SO}(N)$ gauge group (with the central node $8 *$ being $\mathrm{SO}(8) / \mathbb{Z}_{2}$ ) and each black node labeled by $M$ denotes a 
$\mathrm{USp}(M)$ gauge group.

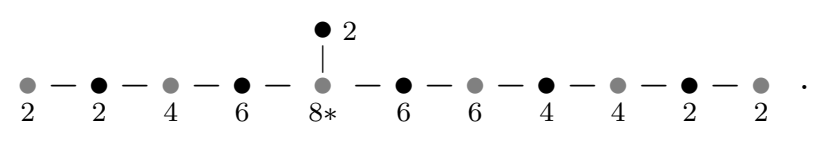

Note that the ranks of all gauge groups add up to 24. This is the quaternionic dimension of the Coulomb branch, which indeed agrees with the dimension of the Higgs branch of the theory of 48 free half-hypermultiplets.

The Coulomb branch Hilbert series of the mirror theory is

$$
\begin{aligned}
& H(t ; \boldsymbol{x}, \boldsymbol{y})=\sum_{a_{1}, a_{2}, a_{3}, a_{4} \geq 0} t^{-\delta_{\mathrm{SO}(8)}(\boldsymbol{n}(\boldsymbol{a}))} P_{\mathrm{SO}(8)}(t ; \boldsymbol{n}(\boldsymbol{a})) H\left[T_{(5,3)}(\mathrm{SO}(8))\right](t ; \boldsymbol{n}(\boldsymbol{a})) \times \\
& H\left[T_{\left(2^{2}, 1^{4}\right)}(\mathrm{SO}(8))\right](t ; \boldsymbol{y} ; \boldsymbol{n}(\boldsymbol{a})) H\left[T_{\left(1^{8}\right)}(\mathrm{SO}(8))\right](t ; \boldsymbol{x} ; \boldsymbol{n}(\boldsymbol{a})),
\end{aligned}
$$

where $\boldsymbol{x}=\left(x_{1}, x_{2}, x_{3}\right)$ and $\boldsymbol{y}=\left(y_{1}, \ldots, y_{4}\right)$ are respectively fugacities of $\mathrm{SU}(2)^{3}$ and $\mathrm{SO}(8)$ symmetries and the function $P_{\mathrm{SO}(8)}$ is defined as in (A.10) of [1], and

$$
\begin{aligned}
\boldsymbol{n}(\boldsymbol{a}) & =\left(a_{1}+a_{2}+\frac{a_{3}+a_{4}}{2}, a_{2}+\frac{a_{3}+a_{4}}{2}, \frac{a_{3}+a_{4}}{2}, \frac{-a_{3}+a_{4}}{2}\right), \\
\delta_{\mathrm{SO}(8)}(\boldsymbol{n}) & =6 n_{1}+4 n_{2}+2 n_{3}, \\
H\left[T_{\left(1^{8}\right)}(\mathrm{SO}(8))\right](t ; \boldsymbol{x} ; \boldsymbol{n}) & =t^{\frac{1}{2} \delta_{\mathrm{SO}(8)}(\boldsymbol{n})}(1-t)^{4} K_{\left(1^{8}\right)}(\boldsymbol{x} ; t) \Psi_{D_{4}}(\boldsymbol{x} ; \boldsymbol{n} ; t), \\
H\left[T_{(5,3)}(\mathrm{SO}(8))\right](t ; \boldsymbol{n}) & =t^{\frac{1}{2} \delta_{\mathrm{SO}(8)}(\boldsymbol{n})}(1-t)^{4} K_{(5,3)}(t) \Psi_{D_{4}}\left(1, t, t^{-1}, t^{2} ; \boldsymbol{n} ; t\right), \\
H\left[T_{\left(2^{2}, 1^{4}\right)}(\mathrm{SO}(8))\right](t ; \boldsymbol{y} ; \boldsymbol{n}) & =t^{\frac{1}{2} \delta_{\mathrm{SO}(8)}(\boldsymbol{n})}(1-t)^{4} K_{\left(2^{2}, 1^{4}\right)}(\boldsymbol{y} ; t) \Psi_{D_{4}}\left(t x_{1}^{-1}, t y_{1}, y_{2}, y_{3} ; \boldsymbol{n} ; t\right), \\
K_{\left(1^{8}\right)}(\boldsymbol{x} ; t) & =\mathrm{PE}\left[\chi_{[0,1,0,0]}^{D_{4}}(\boldsymbol{x}) t\right], \\
K_{(5,3)}(t) & =\mathrm{PE}\left[3 t^{2}+t^{3}+2 t^{4}\right], \\
K_{\left(2^{2}, 1^{4}\right)}(t ; \boldsymbol{y}) & =\mathrm{PE}\left[t\left(2+\chi_{[2]}^{\mathrm{SU}(2)}\left(y_{1}\right)+\chi_{[2]}^{\mathrm{SU}(2)}\left(y_{2}\right) \chi_{[2]}^{\mathrm{SU}(2)}\left(y_{3}\right)\right)\right. \\
& \left.+t^{3 / 2} \chi_{[2]}^{\mathrm{SU}(2)}\left(y_{1}\right)\left\{\chi_{[2]}^{\mathrm{SU}(2)}\left(y_{2}\right)+\chi_{[2]}^{\mathrm{SU}(2)}\left(y_{3}\right)\right\}+t^{2}\right] .
\end{aligned}
$$

It can be checked that the first few terms in the power series of (4.7) agrees with

$$
\begin{aligned}
H(t ; \boldsymbol{x}, \boldsymbol{y})= & \operatorname{PE}\left[\left\{\chi_{[1,0,0,0]}^{D_{4}}(\boldsymbol{x}) \chi_{[1]}^{\mathrm{SU}(2)}\left(y_{1}\right)+\chi_{[0,0,1,0]}^{D_{4}}(\boldsymbol{x}) \chi_{[1]}^{\mathrm{SU}(2)}\left(y_{2}\right)\right.\right. \\
& \left.\left.+\chi_{[0,0,0,1]}^{D_{4}}(\boldsymbol{x}) \chi_{[1]}^{\mathrm{SU}(2)}\left(y_{3}\right)\right\} t\right]
\end{aligned}
$$

namely the Hilbert series of 48 free half-hypermultiplets, as expected from mirror symmetry.

\subsection{2 $D_{4}$ punctures: $\left(3^{2}, 1^{2}\right),\left(2^{2}, 1^{4}\right)$ and $\left(2^{2}, 1^{4}\right)$}

The quiver diagram of the mirror of this Sicilian theory can be obtained by gluing the quiver diagrams of $T_{\left(3^{2}, 1^{2}\right)}(\mathrm{SO}(8)), T_{\left(2^{2}, 1^{4}\right)}(\mathrm{SO}(8))$ and $T_{\left(2^{2}, 1^{4}\right)}(\mathrm{SO}(8))$ via the common symmetry $\mathrm{SO}(8) / \mathbb{Z}_{2}$; this is depicted in (4.10), where each gray node labeled by $N$ denotes an $\mathrm{SO}(N)$ gauge group (with the central node $8 *$ being $\mathrm{SO}(8) / \mathbb{Z}_{2}$ ) and each black node labeled by $M$ 
denotes a $\operatorname{USp}(M)$ gauge group.

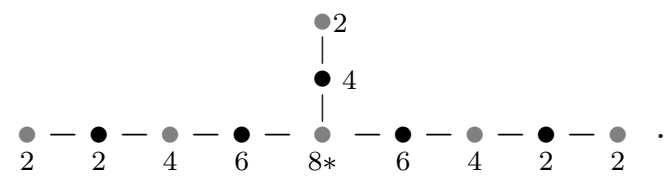

The quaternionic dimension of the Coulomb branch of this theory, equal to the sum of the ranks of all gauge groups, is 21 .

The global symmetries associated with these punctures are respectively $\mathrm{SO}(2)^{2}, \mathrm{SU}(2)^{3}$ and $\mathrm{SU}(2)^{3}$. According to page 28 of [26], this Sicilian theory can be identified with the $T_{4}$ theory and the global symmetry enhances to $\mathrm{SU}(4)^{3}$. Indeed, the Higgs branch of the $T_{4}$ theory is 21 quaternionic dimensional; this is in agreement with the dimension of the Coulomb branch of the mirror theory.

The Coulomb branch Hilbert series of theory depicted in (4.10) is

$$
\begin{aligned}
H(t ; \boldsymbol{x}, \boldsymbol{y}, \boldsymbol{z})= & \sum_{a_{1}, a_{2}, a_{3}, a_{4} \geq 0} t^{-\delta_{\mathrm{SO}(8)}(\boldsymbol{n}(\boldsymbol{a}))} P_{\mathrm{SO}(8)}(t ; \boldsymbol{n}(\boldsymbol{a})) H\left[T_{\left(3^{2}, 1^{2}\right)}(\mathrm{SO}(8))\right](t ; \boldsymbol{z} ; \boldsymbol{n}(\boldsymbol{a})) \times \\
& H\left[T_{\left(2^{2}, 1^{4}\right)}(\mathrm{SO}(8))\right](t ; \boldsymbol{y} ; \boldsymbol{n}(\boldsymbol{a})) H\left[T_{\left(2^{2}, 1^{4}\right)}(\mathrm{SO}(8))\right](t ; \boldsymbol{x} ; \boldsymbol{n}(\boldsymbol{a})),
\end{aligned}
$$

where $\boldsymbol{x}=\left(x_{1}, x_{2}, x_{3}\right)$ and $\boldsymbol{y}=\left(y_{1}, y_{2}, y_{3}\right)$ are fugacities for $\mathrm{SU}(2)^{3}, \boldsymbol{z}=\left(z_{1}, z_{2}\right)$ are fugacities for $\mathrm{SO}(2)^{2}$, and

$$
\begin{aligned}
H\left[T_{\left(3^{2}, 1^{2}\right)}(\mathrm{SO}(8))\right](t ; \boldsymbol{z} ; \boldsymbol{n}) & =t^{\frac{1}{2} \delta_{\mathrm{SO}(8)}(\boldsymbol{n})}(1-t)^{4} K_{\left(3^{2}, 1^{2}\right)}(\boldsymbol{z} ; t) \Psi_{D_{4}}\left(z_{1} t, z_{1} t^{-1}, z_{1}, z_{2} ; \boldsymbol{n} ; t\right), \\
K_{\left(3^{2}, 1^{2}\right)}(\boldsymbol{z} ; t) & =\mathrm{PE}\left[2 t+\left(z_{1}^{2}+1+z_{1}^{-2}+\sum_{\epsilon_{1}, \epsilon_{2}= \pm 1} z_{1}^{\epsilon_{1}} z_{2}^{\epsilon_{2}}\right) t^{2}+t^{3}\right] .
\end{aligned}
$$

Computing the power series in $t$ of the above expression (4.11), we find that at order $t$, the 45 gauge invariants transform as follows:

$$
\begin{aligned}
& \left(z_{1}+z_{1}^{-1}\right)[1]_{x_{1}}[1]_{y_{1}}+[2]_{x_{1}}+[2]_{y_{1}}+1 \\
& +\left(z_{1}^{1 / 2} z_{2}^{1 / 2}+z_{1}^{-1 / 2} z_{2}^{-1 / 2}\right)[1]_{x_{2}}[1]_{y_{2}}+[2]_{x_{2}}+[2]_{y_{2}}+1 \\
& +\left(z_{1}^{1 / 2} z_{2}^{-1 / 2}+z_{1}^{-1 / 2} z_{2}^{1 / 2}\right)[1]_{x_{3}}[1]_{y_{3}}+[2]_{x_{3}}+[2]_{y_{3}}+1
\end{aligned}
$$

where $[\cdots]_{\boldsymbol{a}}$ denotes the character of representation $[\cdots]$ written in terms of $\boldsymbol{a}$. Note that each line gives the decomposition of the adjoint representation of $\mathrm{SU}(4)$ in terms of representations of $\mathrm{SO}(2) \times \mathrm{SU}(2)^{2}$. Hence these 45 generators indeed decompose into three copies of 15, each transforming in the adjoint representation of an $\mathrm{SU}(4)$ in $\mathrm{SU}(4)^{3}$.

A similar analysis can be performed at higher orders of $t$. Moreover, the unrefined Hilbert series, i.e. all $x_{i}, y_{i}, z_{i}$ are set to 1 , can be computed from (4.11):

$$
H(t ; \mathbf{1}, \mathbf{1}, \mathbf{1})=1+45 t+128 t^{3 / 2}+1249 t^{2}+5504 t^{5 / 2}+\ldots ;
$$

the result is in agreement with [4]. 


\subsection{3 $D_{4}$ punctures: $(5,3),(5,3),\left(2^{4}\right)$ and $\left(3,1^{5}\right)$}

The quiver diagram of the mirror of this Sicilian theory can be obtained by gluing the quiver diagrams of $T_{(5,3)}(\mathrm{SO}(8)), T_{(5,3)}(\mathrm{SO}(8)), T_{\left(2^{4}\right)}(\mathrm{SO}(8))$ and $T_{\left(3,1^{5}\right)}(\mathrm{SO}(8))$ via the common symmetry $\mathrm{SO}(8) / \mathbb{Z}_{2}$; this is depicted in (4.15), where each gray node labeled by $N$ denotes an $\mathrm{SO}(N)$ gauge group (with the central node $8 *$ being $\mathrm{SO}(8) / \mathbb{Z}_{2}$ ) and each black node labeled by $M$ denotes a $\operatorname{USp}(M)$ gauge group.

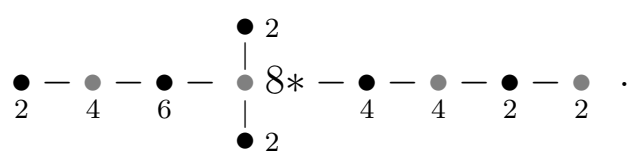

The quaternionic dimension of the Coulomb branch of this theory, equal to the sum of the the ranks of all gauge groups, is 18 .

The global symmetries associated with each puncture are respectively trivial, trivial, $\mathrm{USp}(4)$ and $\mathrm{SO}(5) \simeq \mathrm{USp}(4)$. According to the top diagram of page 32 of [26], this Sicilian theory can be identified with the $G_{2}$ gauge theory with 4 fundamental hypermultiplets and 4 free hypermultiplets, which has $\operatorname{USp}(8)$ flavor symmetry. Indeed, the quaternionic dimension of the Higgs branch of this theory is equal to $\frac{1}{2}(7 \times 4)+4=18$; this is in agreement with the dimension of the Coulomb branch of the mirror theory.

The Higgs branch Hilbert series of $G_{2}$ gauge theory with 4 flavors of fundamental hypers, plus 4 free hypers. In the following, we write

$$
\tau=t^{1 / 2}
$$

The $F$-flat Hilbert series is given by

$$
\mathcal{F}^{b}(\tau ; \boldsymbol{z} ; \boldsymbol{x})=\mathrm{PE}\left[\tau \chi_{[1,0,0,0]}^{\mathrm{USp}(8)}(\boldsymbol{x})\right] \times \operatorname{PE}\left[\tau \chi_{[1,0,0,0]}^{\mathrm{USp}(8)}(\boldsymbol{x}) \chi_{[1,0]}^{G_{2}}(\boldsymbol{z})-\tau^{2} \chi_{[0,1]}^{G_{2}}(\boldsymbol{z})\right] .
$$

The Higgs branch Hilbert series can be obtained by integrating over the $G_{2}$ gauge group as follows:

$$
g(\tau, \boldsymbol{x})=\int \mathrm{d} \mu_{G_{2}}(\boldsymbol{z}) \mathcal{F}^{b}(\tau ; \boldsymbol{z} ; \boldsymbol{x})
$$

where the Haar measure of $G_{2}$ is given by

$$
\begin{aligned}
\int \mathrm{d} \mu_{G_{2}}(\boldsymbol{z})= & \frac{1}{(2 \pi i)^{2}} \oint_{\left|z_{1}\right|=1} \frac{\mathrm{d} z_{1}}{z_{1}} \oint_{\left|z_{2}\right|=1} \frac{\mathrm{d} z_{2}}{z_{2}}\left(1-z_{1}\right)\left(1-z_{1}^{2} z_{2}^{-1}\right)\left(1-z_{1}^{3} z_{2}^{-1}\right) \\
& \left(1-z_{2}\right)\left(1-z_{2} z_{1}^{-1}\right)\left(1-z_{2}^{2} z_{1}^{-3}\right)
\end{aligned}
$$

The first few terms in the power series of the Higgs branch Hilbert series $g(\tau, \boldsymbol{x})$ are

$$
\begin{aligned}
g(\tau, \boldsymbol{x})= & \operatorname{PE}\left[\tau \chi_{[1,0,0,0]}^{C_{4}}(\boldsymbol{x})\right] \times\left\{1+\chi_{[2,0,0,0]}^{C_{4}}(\boldsymbol{x}) \tau^{2}+\left(\chi_{[1,0,0,0]}^{C_{4}}(\boldsymbol{x})+\chi_{[0,0,1,0]}^{C_{4}}(\boldsymbol{x})\right) \tau^{3}\right. \\
& \left.+\left(\chi_{[4,0,0,0]}^{C_{4}}(\boldsymbol{x})+\chi_{[0,1,0,0]}^{C_{4}}(\boldsymbol{x})+\chi_{[0,2,0,0]}^{C_{4}}(\boldsymbol{x})+\chi_{[0,0,0,1]}^{C_{4}}(\boldsymbol{x})+1\right) \tau^{4}+\ldots\right\} .
\end{aligned}
$$

Below we reproduce this Hilbert series from the Coulomb branch of the mirror theory of this Sicilian theory. 
The Coulomb branch Hilbert series of the mirror theory. The Coulomb branch Hilbert series is given by

$$
\begin{aligned}
H(t ; \boldsymbol{x}, \boldsymbol{y})= & \sum_{a_{1}, a_{2}, a_{3}, a_{4} \geq 0} t^{-\delta_{\mathrm{SO}(8)}(\boldsymbol{n}(\boldsymbol{a}))} P_{\mathrm{SO}(8)}(t ; \boldsymbol{n}(\boldsymbol{a})) H\left[T_{(5,3)}(\mathrm{SO}(8))\right](t ; \boldsymbol{n}(\boldsymbol{a})) \times \\
& H\left[T_{(5,3)}(\mathrm{SO}(8))\right](t ; \boldsymbol{n}(\boldsymbol{a})) H\left[T_{\left(2^{4}\right)}(\mathrm{SO}(8))\right](t ; \boldsymbol{y} ; \boldsymbol{n}(\boldsymbol{a})) \times \\
& H\left[T_{\left(3,1^{5}\right)}(\mathrm{SO}(8))\right](t ; \boldsymbol{x} ; \boldsymbol{n}(\boldsymbol{a})),
\end{aligned}
$$

where $\boldsymbol{x}=\left(x_{1}, x_{2}\right)$ and $\boldsymbol{y}=\left(y_{1}, y_{2}\right)$ are fugacities for $\mathrm{SO}(5)$ and $\mathrm{USp}(4)$ respectively, and

$$
\begin{aligned}
H\left[T_{\left(3,1^{5}\right)}(\mathrm{SO}(8))\right](t ; \boldsymbol{x} ; \boldsymbol{n}) & =t^{\frac{1}{2} \delta_{\mathrm{SO}(8)}(\boldsymbol{n})}(1-t)^{4} K_{\left(3,1^{5}\right)}(\boldsymbol{x} ; t) \Psi_{D_{4}}\left(1, t, x_{1}, x_{2} ; \boldsymbol{n} ; t\right), \\
K_{\left(3,1^{5}\right)}(\boldsymbol{x} ; t) & =\mathrm{PE}\left[t \chi_{[0,2]}^{B_{2}}(\boldsymbol{x})+t^{2}\left(1+\chi_{[1,0]}^{B_{2}}(\boldsymbol{x})\right] .\right.
\end{aligned}
$$

We have checked that the first few terms in the power series of this Hilbert series agree with (4.20). In particular, the unrefined Hilbert series is

$$
\begin{aligned}
H\left[T_{\left(3,1^{5}\right)}(\mathrm{SO}(8))\right](t, \boldsymbol{x}=\mathbf{1}, \boldsymbol{y}=1) & =1+8 t^{1 / 2}+72 t+464 t^{3 / 2}+2782 t^{2}+\ldots \\
& =\frac{1}{\left(1-t^{1 / 2}\right)^{8}}\left(1+36 t+56 t^{3 / 2}+708 t^{2}+\ldots\right) .
\end{aligned}
$$

\section{Coulomb branch Hilbert series of $3 d$ theories with tri-vertices}

In this section we consider the Coulomb branch of theories on two M5-branes compactified on a Riemann surface with punctures times a circle of vanishing size. The latter are referred to as $3 d \mathrm{SU}(2)$ Sicilian theories [6, 23], or $3 d$ theories with tri-vertices [11]. We emphasize that in this section we aim to compute the Coulomb branch Hilbert series of tri-vertex theories, in contrast to section 3.1.1 in which we considered the Coulomb branch of their mirrors.

We follow the notation adopted in [11]. The Lagrangian of a tri-vertex theory is specified by a graph made of tri-valent vertices connected by lines. Each line denotes an $\mathrm{SU}(2)$ group; an internal line (of finite length) denotes a gauge group, whereas an external line (of infinite length) denotes a flavor group. Each vertex denotes 8 half-hypermultiplets in the tri-fundamental representation of the corresponding $\mathrm{SU}(2)^{3}$ group. Such graphs are classified topologically by the genus $g$ and the number $e$ of external legs. It was found in [11] that the Higgs branch of such theories depends only on $g$ and $e$ and not on the details of how the vertices are connected to each other.

In this section we focus only on the cases with $g=0$, i.e. tree diagrams, since for higher genus the theory is bad. For $g=0$, the Coulomb branch Hilbert series can be evaluated explicitly and it depends only on the number of external legs $e$ and not on the details of the graph. In section 5.3 we present certain generating functions and recursive formulae that serve as powerful tools for computing Hilbert series of these class of theories using gluing techniques. The fact that such generating functions depend solely on the number of external legs $e$ is proven in section 5.3.2.

It would be interesting to understand how to compute the Coulomb branch Hilbert series of theories with higher genus by determining whether they flow to a good theory in the IR. 


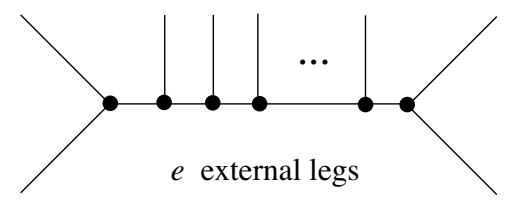

Figure 6. A tri-vertex diagram with genus zero and $e$ external legs. The number of gauge groups is $e-3$.

\subsection{The case of $g=0$}

We consider the Coulomb branch of $3 d \mathcal{N}=4$ gauge theories based on tri-vertex tree $(g=0)$ diagrams, with $\mathrm{SU}(2)$ gauge groups associated to internal edges and tri-fundamental halfhypermultiplets associated to nodes.

We will see in a few examples that, as for the Higgs branch [11], the Coulomb branch only depends on the number of external edges; see an example for $g=0$ and $e=6$ in section 5.1.2 below. We give a general proof of this fact in subsection 5.3.2.

\subsubsection{General formula for $g=0$ and any $e$}

In the following we restrict to linear diagrams where each tri-vertex has one external leg, except for those at the ends of the line which have two external legs; see figure 6 .

Let us consider $e=n+3$ external legs. The gauge group is $\mathrm{SU}(2)^{n}$. The Hilbert series of the Coulomb branch of this gauge theory is

$$
H[g=0, e=n+3](t)=\sum_{a_{1}=0}^{\infty} \cdots \sum_{a_{n}=0}^{\infty} t^{\Delta(a)} \prod_{i=1}^{n} P_{\mathrm{SU}(2)}\left(t ; a_{i}\right) .
$$

The dimension formula for monopole operators is

$$
\begin{aligned}
\Delta(\boldsymbol{a}) & =\frac{1}{2}\left[2\left(\left|a_{1}\right|+\left|-a_{1}\right|\right)+\sum_{s_{1,2}=0}^{1} \sum_{j=1}^{n-1}\left|(-1)^{s_{1}} a_{j}+(-1)^{s_{2}} a_{j+1}\right|+2\left(\left|a_{n}\right|+\left|-a_{n}\right|\right)\right] \\
-\sum_{i=1}^{n}\left|2 a_{i}\right| & =-2 \sum_{i=2}^{n-1}\left|a_{i}\right|+\sum_{i=1}^{n-1}\left(\left|a_{i}-a_{i+1}\right|+\left|a_{i}+a_{i+1}\right|\right),
\end{aligned}
$$

where $a_{i}, i=1, \ldots, n$ are the GNO charges in the weight lattice of the GNO dual $\mathrm{SO}(3)^{n}$ group: $a_{i} \in \mathbb{Z}_{\geq 0}$. The classical factor accounts for the Casimir invariants of the residual gauge group which is not broken by the monopole flux. For an $\mathrm{SU}(2)$ gauge group, the classical factor is

$$
P_{\mathrm{SU}(2)}(t ; a)=\left\{\begin{array}{ll}
\frac{1}{1-t^{2}}, & a=0 \\
\frac{1}{1-t}, & a>0
\end{array} .\right.
$$




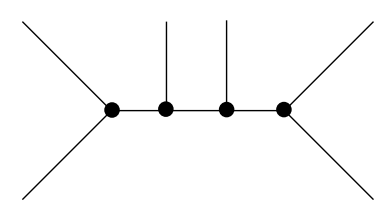

(a)

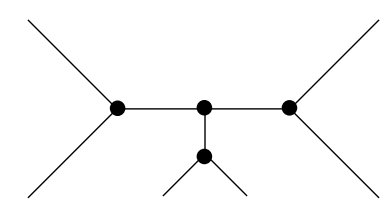

(b)

Figure 7. Two tri-vertex diagrams with genus zero and 6 external legs.

The result for the Hilbert series (5.1) appears to be

$$
\begin{aligned}
H[g=0, e=n+3](t) & =\frac{\sum_{j=0}^{n}\left(\begin{array}{c}
n \\
j
\end{array}\right)\left[\left(\begin{array}{c}
n \\
j
\end{array}\right) t^{2 j}-\left(\begin{array}{c}
n \\
j+1
\end{array}\right) t^{2 j+1}\right]}{(1-t)^{2 n}(1+t)^{n}} \\
& =\frac{1}{(1-t)^{2 n}(1+t)^{n}}\left[-n t_{2} F_{1}\left(1-n,-n ; 2 ; t^{2}\right)+{ }_{2} F_{1}\left(-n,-n ; 2 ; t^{2}\right)\right] .
\end{aligned}
$$

\subsubsection{Special case of $g=0$ and $e=6$}

As an example of the fact that the Coulomb branch only depends on the number of external edges we consider the case $e=6$. There are two diagrams corresponding to $g=0$ and $e=6$, depicted in figure 7 .

Diagram (a). The Coulomb branch Hilbert series of diagram (a) is given by (5.4):

$$
H_{(a)}(t)=\frac{1-3 t+9 t^{2}-9 t^{3}+9 t^{4}-3 t^{5}+t^{6}}{(1-t)^{6}(1+t)^{3}} .
$$

Diagram (b). For diagram (b), we have

$$
\Delta_{(b)}(\boldsymbol{a})=\frac{1}{2}\left[\frac{1}{2} \sum_{s_{1,2,3}=0}^{1}\left|\sum_{i=1}^{3}(-1)^{s_{i}} a_{i}\right|+2 \sum_{i=1}^{3}\left(\left|a_{i}\right|+\left|-a_{i}\right|\right)\right]-\sum_{i=1}^{3}\left|2 a_{i}\right|,
$$

Observe that this is not equal to $\Delta_{(a)}(\boldsymbol{a})$ which is given in (5.2). However, the Hilbert series of the Coulomb branch is given by

$$
\begin{aligned}
H_{(b)}(t) & =\sum_{a_{1}, a_{2}, a_{3}=0}^{\infty} t^{\Delta_{(b)}(\boldsymbol{a})} \prod_{i=1}^{3} P_{\mathrm{SU}(2)}\left(t ; a_{i}\right) \\
& =\frac{1-3 t+9 t^{2}-9 t^{3}+9 t^{4}-3 t^{5}+t^{6}}{(1-t)^{6}(1+t)^{3}}=H_{(a)}(t),
\end{aligned}
$$

which is indeed equal to that of diagram (a).

\subsection{Turning on background fluxes}

So far we have computed the Coulomb Hilbert series without considering the background monopole charges coming from the global symmetries of the theory. In this section, we turn on such background charges for the flavor symmetries present in the theory and the 
corresponding Hilbert series will, of course, depend on such charges. This will turn out to be extremely useful in subsequent computations.

Let us first consider the $T_{2}$ theory $(g=0, e=3)$. The Coulomb branch Hilbert series with background fluxes turned on is simply

$$
H\left[T_{2}\right]\left(t ; a_{1}, a_{2}, a_{3}\right)=t^{\Delta_{g=0, e=3}\left(a_{1}, a_{2}, a_{3}\right)},
$$

where $a_{1}, a_{2}, a_{3} \geq 0$ are the background fluxes and

$$
\Delta_{g=0, e=3}\left(a_{1}, a_{2}, a_{3}\right)=\frac{1}{2}\left[\frac{1}{2} \sum_{s_{1,2,3}=0}^{1}\left|(-1)^{s_{1}} a_{1}+(-1)^{s_{2}} a_{2}+(-1)^{s_{3}} a_{3}\right|\right] .
$$

The Coulomb branch Hilbert series with background fluxes turned on can be handled more easily if we introduce extra fugacities to keep track of such background charges. In this way, we end up computing the generating function of the Coulomb branch Hilbert series. This is the topic of the next section.

\subsection{Generating functions of Coulomb branch Hilbert series}

For a theory with genus zero and $e$ external legs, we can construct a generating function

$$
G_{e}\left(z_{1}, \ldots, z_{e}\right)=\sum_{a_{1}=0}^{\infty} \cdots \sum_{a_{e}=0}^{\infty} H[e]\left(t ; a_{1}, \ldots, a_{e}\right) \prod_{i=1}^{e} z_{i}^{a_{i}}
$$

where $a_{1}, \ldots, a_{e}$ are the background fluxes for the $\mathrm{SU}(2)^{e}$ global symmetry group associated to the external legs and $H[e]\left(t ; a_{1}, \ldots, a_{e}\right)$ is the usual Hilbert series with these background fluxes turned on. Note that we omit the $t$ dependence in $G_{e}\left(z_{1}, \ldots, z_{e}\right)$ for the sake of brevity. To turn off the background fluxes, we simply set all $z_{i}$ to zero:

$$
H[e](t ; 0, \ldots, 0)=G_{e}(0,0, \ldots, 0) .
$$

We go over the computations of generating functions in the examples below.

The $\boldsymbol{T}_{\mathbf{2}}$ theory. From (5.8), we have

$$
G_{e=3}\left(z_{1}, z_{2}, z_{3}\right)=\sum_{a_{1}=0}^{\infty} \sum_{a_{2}=0}^{\infty} \sum_{a_{3}=0}^{\infty} t^{\Delta_{e=3}(\boldsymbol{a})} z_{1}^{a_{1}} z_{2}^{a_{2}} z_{3}^{a_{3}},
$$

Evaluating the summations, we obtain

$$
\begin{aligned}
G_{e=3}(\boldsymbol{z})= & \frac{1}{\prod_{i=1}^{3}\left(1-t^{2} z_{i}\right) \prod_{1 \leq j<k \leq 3}\left(1-t^{2} z_{j} z_{k}\right)} \times\left[1+z_{1} z_{2} z_{3} t^{3}\right. \\
& +\left(-z_{1} z_{2}-z_{1} z_{3}-z_{2} z_{3}-3 z_{1} z_{2} z_{3}\right) t^{4}+\left(-z_{1}^{2} z_{2} z_{3}-z_{1} z_{2}^{2} z_{3}-z_{1} z_{2} z_{3}^{2}\right) t^{5} \\
& +2\left(z_{1} z_{2} z_{3}+z_{1}^{2} z_{2} z_{3}+z_{1} z_{2}^{2} z_{3}+z_{1} z_{2} z_{3}^{2}\right) t^{6}+\left(z_{1}^{2} z_{2}^{2} z_{3}+z_{1}^{2} z_{2} z_{3}^{2}+z_{1} z_{2}^{2} z_{3}^{2}\right) t^{7} \\
& \left.+\left(-z_{1}^{2} z_{2}^{2} z_{3}-z_{1}^{2} z_{2} z_{3}^{2}-z_{1} z_{2}^{2} z_{3}^{2}\right) t^{8}-z_{1}^{2} z_{2}^{2} z_{3}^{2} t^{9}\right] .
\end{aligned}
$$

Observe that $G_{e=3}(\boldsymbol{z})$ is invariant under the permutations of $z_{1}, z_{2}, z_{3}$. Upon setting $z_{1}=$ $z_{2}=z_{3}=0$, we recover the (trivial) Hilbert series of the Coulomb branch as expected:

$$
G_{e=3}(0,0,0)=1 .
$$




\subsubsection{Gluing generating functions and recursive formula}

If we glue a tree diagram with $e_{1}$ external legs with another tree diagram with $e_{2}$ external legs via an external leg, the resulting diagram is a tree diagram of $e_{1}+e_{2}-2$ external legs. In terms of the Coulomb branch Hilbert series, this gluing operation can be formulated as

$$
\begin{aligned}
H\left[e_{1}\right. & \left.+e_{2}-2\right](\boldsymbol{a}) \\
& =\sum_{a=0}^{\infty} H\left[e_{1}\right]\left(a_{1}, \ldots, a_{e_{1}-1}, a\right) P_{\mathrm{SU}(2)}(t ; a) t^{-2 a} H\left[e_{2}\right]\left(a, a_{e_{1}}, \ldots, a_{e_{1}+e_{2}-2}\right)
\end{aligned}
$$

where in this formula we glue the $e_{1}$-th external leg of the first diagram with the first leg of the second diagram. In terms of the generating functions, we have

$$
\begin{aligned}
G_{e_{1}+e_{2}-2}(\boldsymbol{a})= & \oint_{|u|=1} \frac{\mathrm{d} u}{2 \pi i u} \oint_{|w|=1} \frac{\mathrm{d} u}{2 \pi i w} \sum_{a=0}^{\infty} G_{e_{1}}\left(z_{1}, \ldots, z_{e_{1}-1}, u\right) \times \\
& u^{-a} P_{\mathrm{SU}(2)}(t ; a) t^{-2 a} w^{-a} G_{e_{2}}\left(w, z_{e_{1}}, \ldots, z_{e_{1}+e_{2}-2}\right)
\end{aligned}
$$

The recursive formula. The diagrams with $g=0$ and $e+1$ external legs can be constructed recursively by gluing the diagram $(g=0, e=3)$ with another diagram with $e$ external legs. We can thus obtain the recursive formula for the generating functions as follows.

From (5.16) we obtain

$$
\begin{aligned}
G_{e+1}(\boldsymbol{z})= & \oint_{|u|=1} \frac{\mathrm{d} u}{2 \pi i u} \oint_{|w|=1} \frac{\mathrm{d} w}{2 \pi i w} \sum_{a=0}^{\infty} G_{e=3}\left(z_{1}, z_{2}, u\right) \times \\
& u^{-a} P_{\mathrm{SU}(2)}(t ; a) t^{-2 a} w^{-a} G_{e}\left(w, z_{3}, \ldots, z_{e+1}\right) .
\end{aligned}
$$

We write the infinite sum as follows:

$$
\sum_{a=0}^{\infty} u^{-a} P_{\mathrm{SU}(2)}(t ; a) t^{-2 a} w^{-a}=-\frac{t}{1-t^{2}}+\frac{t^{2} u w}{(1-t)\left(t^{2} u w-1\right)}
$$

Thus we have

$$
\begin{aligned}
G_{e+1}(\boldsymbol{z})= & -\frac{t}{1-t^{2}} \oint_{|u|=1} \frac{\mathrm{d} u}{2 \pi i u} \oint_{|w|=1} \frac{\mathrm{d} w}{2 \pi i w} G_{e=3}\left(z_{1}, z_{2}, u\right) G_{e}\left(w, z_{3}, \ldots, z_{e+1}\right) \\
& +\frac{t^{2}}{1-t} \oint_{|u|=1} \frac{\mathrm{d} u}{2 \pi i} \oint_{|w|=1} \frac{\mathrm{d} w}{2 \pi i} \frac{G_{e=3}\left(z_{1}, z_{2}, u\right) G_{e}\left(w, z_{3}, \ldots, z_{e+1}\right)}{t^{2} u w-1} \\
= & -\frac{t}{1-t^{2}} G_{e=3}\left(z_{1}, z_{2}, 0\right) G_{e}\left(0, z_{3}, \ldots, z_{e+1}\right)+ \\
& +\frac{1}{1-t} \oint_{|w|=1} \frac{\mathrm{d} w}{2 \pi i} \frac{G_{e=3}\left(z_{1}, z_{2}, w^{-1} t^{-2}\right) G_{e}\left(w, z_{3}, \ldots, z_{e+1}\right)}{w}
\end{aligned}
$$


In the integral of the last line, we see from (5.13) that the poles of $G_{e=3}\left(z_{1}, z_{2}, w^{-1} t^{-2}\right)$ are at $w=1, w=z_{1}$ and $w=z_{2}$. Using the residue theorem, we obtain

$$
\begin{aligned}
G_{e+1}(\boldsymbol{z})= & -\frac{t}{1-t^{2}} G_{e=3}\left(z_{1}, z_{2}, 0\right) G_{e}\left(0, z_{3}, \ldots, z_{e+1}\right)+ \\
& +\frac{1}{(1-t)} \operatorname{Res}\left[G_{e=3}\left(z_{1}, z_{2}, w^{-1} t^{-2}\right) ; w=1\right] G_{e}\left(1, z_{3}, \ldots, z_{e+1}\right) \\
& +\frac{1}{(1-t) z_{1}} \operatorname{Res}\left[G_{e=3}\left(z_{1}, z_{2}, w^{-1} t^{-2}\right) ; w=z_{1}\right] G_{e}\left(z_{1}, z_{3}, \ldots, z_{e+1}\right) \\
& +\frac{1}{(1-t) z_{2}} \operatorname{Res}\left[G_{e=3}\left(z_{1}, z_{2}, w^{-1} t^{-2}\right) ; w=z_{2}\right] G_{e}\left(z_{2}, z_{3}, \ldots, z_{e+1}\right) .
\end{aligned}
$$

Using (5.13), we find that the above residues can be written in terms of simple rational functions:

$$
\begin{aligned}
\operatorname{Res}\left[G_{e=3}\left(z_{1}, z_{2}, w^{-1} t^{-2}\right) ; w=1\right] & =\frac{1}{\left(1-z_{1}\right)\left(1-z_{2}\right)}, \\
\operatorname{Res}\left[G_{e=3}\left(z_{1}, z_{2}, w^{-1} t^{-2}\right) ; w=z_{1}\right] & =\frac{(1-t) z_{1}^{2}\left(-z_{1}-t z_{1}+t z_{2}-t z_{1} z_{2}+t^{2} z_{1} z_{2}+t^{3} z_{1}^{2} z_{2}\right)}{\left(z_{1}-z_{2}\right)\left(1-z_{1}\right)\left(1-t^{2} z_{1}\right)\left(1-t^{2} z_{1} z_{2}\right)}, \\
\operatorname{Res}\left[G_{e=3}\left(z_{1}, z_{2}, w^{-1} t^{-2}\right) ; w\right. & \left.=z_{1}\right]=\frac{(1-t) z_{2}^{2}\left(-z_{2}+t z_{1}-t z_{2}-t z_{1} z_{2}+t^{2} z_{1} z_{2}+t^{3} z_{1} z_{2}^{2}\right)}{\left(z_{2}-z_{1}\right)\left(1-z_{2}\right)\left(1-t^{2} z_{2}\right)\left(1-t^{2} z_{1} z_{2}\right)} .
\end{aligned}
$$

We can thus rewrite (5.20) as

$$
\begin{aligned}
G_{e+1}(\boldsymbol{z})= & -\frac{t\left(1-t^{4} z_{1} z_{2}\right)}{\left(1-t^{2}\right)\left(1-t^{2} z_{1}\right)\left(1-t^{2} z_{2}\right)\left(1-t^{2} z_{1} z_{2}\right)} G_{e}\left(0, z_{3}, \ldots, z_{e+1}\right) \\
& +\frac{1}{(1-t)\left(1-z_{1}\right)\left(1-z_{2}\right)} G_{e}\left(1, z_{3}, \ldots, z_{e+1}\right) \\
& +\left\{\frac{z_{1}\left(-z_{1}-t z_{1}+t z_{2}-t z_{1} z_{2}+t^{2} z_{1} z_{2}+t^{3} z_{1}^{2} z_{2}\right)}{\left(z_{1}-z_{2}\right)\left(1-z_{1}\right)\left(1-t^{2} z_{1}\right)\left(1-t^{2} z_{1} z_{2}\right)} G_{e}\left(z_{1}, z_{3}, \ldots, z_{e+1}\right)\right. \\
& \left.+\left(z_{1} \leftrightarrow z_{2}\right)\right\} .
\end{aligned}
$$

The special case of $z_{2}=\cdots=z_{e+1}=0$. In this case, let us denote

$$
\widehat{G}_{e}(z):=G_{e}(z, 0, \ldots, 0) .
$$

It is immediate from (5.22) that

$$
\begin{aligned}
\widehat{G}_{e+1}(z)= & -\frac{t}{\left(1-t^{2}\right)\left(1-t^{2} z\right)} \widehat{G}_{e}(0)+\frac{1}{(1-t)(1-z)} \widehat{G}_{e}(1) \\
& -\frac{(1+t) z}{(1-z)\left(1-t^{2} z\right)} \widehat{G}_{e}(z) .
\end{aligned}
$$

The ordinary Hilbert series without background fluxes is obtained from $\widehat{G}_{e}(z)$ by setting $z=0$ :

$$
H[e](t)=\widehat{G}_{e}(0) .
$$

Hence one can use the recurrence relation (5.24) to check the exact result (5.4). 


\subsubsection{Proof of the symmetry of the generating functions $G_{e}(z)$}

The Coulomb branch Hilbert series of the tri-vertex theories only depends on the number of external legs. This follows from the fact that $G_{e}\left(z_{1}, \cdots, z_{e}\right)$ is a symmetric function of the variables $z_{1}, \cdots, z_{e}$. In this section we sketch a proof of this statement.

The proof goes trough various steps.

1. We have seen in (5.13) that $G_{e=3}\left(z_{1}, z_{2}, z_{3}\right)$ is invariant under permutations of $z_{1}, z_{2}$, $z_{3}$. Using (5.13) and the recursion relation (5.22) we can evaluate $G_{e=4}\left(z_{1}, z_{2}, z_{3}, z_{4}\right)$, whose expression is too long to be reported here, and explicitly check that it is invariant under permutations of $z_{1}, z_{2}, z_{3}, z_{4}$.

2. We next analyze linear tree-level theories consisting of a linear chain of $e-2$ vertices, each connected to the following one by an internal line, and with a total number of $e$ external legs. An example for the case $e=6$ is given in part (a) of figure 7 . We now show that the generating function $G_{e}\left(z_{1}, \cdots, z_{e}\right)$ for a linear theory is fully symmetric in the $z_{i}$. It is enough to show that it is invariant under the exchange of any pair of neighboring external legs. Let $z_{i}$ and $z_{i+1}$ the fugacities associated with the pair of external legs. We can always obtain the linear theory by gluing a $e=4$ tree diagram containing the two external legs $z_{i}$ and $z_{i+1}$ with two linear theories with $i$ and $e-i$ external legs and write

$$
\begin{aligned}
G_{e}\left(z_{1}, \cdots, z_{e}\right)= & \sum_{a, a^{\prime}=0}^{\infty} G_{i}\left(z_{1}, \cdots, z_{i-1}, a\right) P_{\mathrm{SU}(2)}(t ; a) t^{-2 a} G_{e=4}\left(a, z_{i}, z_{i+1}, a^{\prime}\right) \\
& P_{\mathrm{SU}(2)}\left(t ; a^{\prime}\right) t^{-2 a^{\prime}} G_{e-i}\left(a^{\prime}, z_{i+2}, \cdots, z_{e}\right),
\end{aligned}
$$

where the symmetry in $z_{i}$ and $z_{i+1}$ is manifest.

3. A generic genus zero tri-vertex theory also contains saturated vertices, i.e. vertices that are connected to three other different vertices by internal lines. We now show that any genus zero diagram can be reduced to a linear one with the same generating function. This will prove our statement for all genus zero theories. As an example we can consider the theory in part (b) of figure 7 . We can recognize that the diagram is obtained by gluing two simple three-vertices $(g=0, e=3)$ with a four-vertex diagram $(g=0, e=4)$, and its generating function can be written as

$$
\begin{aligned}
G_{e=6}\left(z_{1}, \cdots, z_{6}\right)= & \sum_{a, a^{\prime}=0}^{\infty} G_{e=3}\left(z_{1}, z_{2}, a\right) P_{\mathrm{SU}(2)}(t ; a) t^{-2 a} G_{e=4}\left(a, a^{\prime}, z_{3}, z_{4}\right) \\
& P_{\mathrm{SU}(2)}\left(t ; a^{\prime}\right) t^{-2 a^{\prime}} G_{e=3}\left(a^{\prime}, z_{5}, z_{6}\right) .
\end{aligned}
$$

Since the four-vertex diagram is fully symmetric under the exchange of the external legs, we can permute them and give a different shape to our diagram. In particular, equation (5.27) is also the generating function for the linear diagram in part (a) of figure 7. In a similar way, whenever a linear diagram is attached to a saturated node by gluing the two external legs at one of its extremities, by permuting its legs we can 
remove the saturated node in favor of a linear structure. By repeating this process many times we can transform any genus zero diagram into a linear one.

This ends our proof. We notice that we can construct the Hilbert series of higher genus tri-vertex theories by identifying external legs of a genus zero graph, adding the appropriate factor $P_{\mathrm{SU}(2)}(t, a)$, the contribution of the gauge fields to the dimension formula and summing over the $a$. Unfortunately, since the resulting theory is bad, the Hilbert series is divergent. We can make it finite by changing the matter content and adding matter fields transforming under the gauge groups of the legs that are identified. For example, by adding one or more adjoint hypermultiplets to each leg that has been identified the Hilbert series becomes convergent. As a curiosity, we notice that the resulting Hilbert series will be fully symmetric under the exchange of the external legs, since the tree-level Hilbert series was. It would be interesting to see if any of these regularized theories are related to the IR behavior of the higher genus tri-vertex theories.

\section{Conclusion}

In this paper we have applied gluing techniques to the computation of the Coulomb branch Hilbert series of mirrors of three dimensional Sicilian theories and we have successfully compared our results with the superconformal index predictions for the Higgs branch of the Sicilian theories themselves. As shown in [4], the Hall-Littlewood limit of the $4 d \mathcal{N}=2$ superconformal index captures the Higgs branch Hilbert series only for genus zero Riemann surfaces. One of the main results of this paper is formula (3.31) for genus zero: it perfectly agrees with the findings of [4], that were obtained in a completely different manner.

We have also computed the Coulomb branch Hilbert series of mirrors of Sicilian theories with genus greater than one. For $N=2$ M5-branes, the Sicilian theories are Lagrangian and their Higgs branch Hilbert series can be computed by standard methods [11]. We have successfully matched those results with our Coulomb branch Hilbert series of the mirror theories, providing a check of our formulas based on mirror symmetry. For $N>2$, there is no other available method for computing the Higgs branch Hilbert series of Sicilian theories. Our results give non-trivial predictions, that would be nice to check in some other way, maybe using the $3 d$ superconformal index.

Our results clarify why the Hall-Littlewood polynomials appear in two different contexts, the Coulomb branch Hilbert series for the $T_{\boldsymbol{\rho}}(G)$ theories and the four dimensional superconformal index of Sicilian theories. It is interesting to see how the Hall-Littlewood limit of the superconformal index formula $[4,10]$, emerging from an apparently unrelated construction, can be naturally reinterpreted in terms of gluing of three dimensional building blocks. It would be interesting to see if these Hilbert series are computed by some auxiliary three or two dimensional topological theories along the lines of [28-30].

It is natural to expect that the gluing prescription discussed in this paper can be generalized to any group $G$, including non-simply laced and exceptional groups, by gluing Coulomb branch Hilbert series $(2.21)$ of $T_{\boldsymbol{\rho}}(G)$ tails via the common centerless symmetry $G / Z(G)$ in the obvious way. This should yield the Coulomb branch Hilbert series for the 
mirror of $5 \mathrm{~d} \mathcal{N}=2$ super Yang-Mills of gauge group $G$ compactified on the punctured Riemann surface. It would be nice to come up with an explicit check for this proposal.

Our results clearly show that gluing is an efficient technique to evaluate the Coulomb branch Hilbert series, once the Hilbert series with background fluxes of the building blocks are explicitly known. It would be interesting to extend our analysis to cover more general classes of building blocks which can be applied to an even wider class of $\mathcal{N}=4$ gauge theories.

\section{Acknowledgments}

We thank Francesco Benini, Nick Halmagyi, Yuji Tachikawa and Alessandro Tomasiello for useful discussions, and the following institutes and workshops for hospitality and partial support: the Galileo Galilei Institute for Theoretical Physics and INFN and the Geometry of Strings and Fields workshop (SC), the Simons Center for Geometry and Physics and the 2013 Summer Workshop, and Chulalongkorn University and the 3rd Bangkok Workshop on High Energy Theory (AH and NM), École Polytechnique and the String Theory Groups of the universities of Rome "Tor Vergata" and of Oviedo (NM). NM is also grateful to Diego Rodríguez-Gómez, Yolanda Lozano, Raffaele Savelli, Jasmina Selmic, Hagen Triendl, Sarah Maupeu and Mario Pelliccioni for their very kind hospitality. We were partially supported by the STFC Consolidated Grant ST/J000353/1 (SC), the EPSRC programme grant EP/K034456/1 (AH), the ERC grant, Short Term Scientific Mission of COST Action MP1210, and World Premier International Research Center Initiative (WPI Initiative), MEXT, Japan (NM), and INFN and the MIUR-FIRB grant RBFR10QS5J "String Theory and Fundamental Interactions" (AZ).

\section{A Mirrors of Sicilian theories with twisted $D$ punctures}

In this appendix, we briefly discuss $3 d$ Sicilian theories with twisted $D$ punctures. A twisted $D_{N}$ puncture can be written in terms of a $C_{N-1}$ partition $\widetilde{\boldsymbol{\rho}}=\left[\widetilde{\rho}_{i}\right]$ with $\sum_{i} \widetilde{\rho}_{i}=2 N-2$ and $r_{k}$ the number of times that part $k$ appears. The global symmetry to this puncture is given by

$$
G_{\widetilde{\boldsymbol{\rho}}}=\prod_{k \text { odd }} \mathrm{USp}\left(r_{k}\right) \times \prod_{k \text { even }} \mathrm{SO}\left(r_{k}\right)
$$

For example, the global symmetry associated with twisted $D_{4}$ puncture $\left(2,1^{4}\right)$ is $\operatorname{USp}(4)$.

A building block of a mirror of Sicilian theories with twisted $D$ punctures is a gauge theory $T_{\widetilde{\boldsymbol{\rho}}}\left(B_{N-1}\right)$, whose quiver diagram, of the type first considered in [25], is given by (4.3) of [3] and (6.5) of [6]. The quiver gauge theory is bad in the sense of [2], therefore the monopole formula for the Coulomb branch Hilbert series diverges. However, according to [3], the Coulomb branch Hilbert series of the infrared CFT is conjectured to be computed by the Hall-Littlewood formula (2.21), which gives

$$
H\left[T_{\widetilde{\boldsymbol{\rho}}}\left(B_{N-1}\right)\right](t ; \boldsymbol{x} ; \boldsymbol{n})=t^{\frac{1}{2} \delta_{B_{N-1}}(\boldsymbol{n})}(1-t)^{N-1} K_{\boldsymbol{\rho}}^{C_{N-1}}(\boldsymbol{x} ; t) \Psi_{C_{N-1}}^{\boldsymbol{n}}(\boldsymbol{a}(t, \boldsymbol{x}) ; t) .
$$

We will assume the validity of this formula in the rest of the appendix. 
Example: $\widetilde{\boldsymbol{\rho}}=\left(\mathbf{2}, \mathbf{1}^{\mathbf{4}}\right)$. For example, given an $\mathrm{SO}(8)$ twisted puncture $\widetilde{\boldsymbol{\rho}}=\left(2,1^{4}\right)$, the corresponding theory is

$$
T_{\left(2,1^{4}\right)}(\mathrm{SO}(7)): \quad[\mathrm{SO}(7)]-(\mathrm{USp}(4))-(O(5))-(\mathrm{USp}(2))-(O(3)) .
$$

Note that such a tail is a typical component in a mirror pair computation of [25].

The Hall-Littlewood formula (A.2) applied to this theory gives the Coulomb branch Hilbert series

$$
H\left[T_{\left(2,1^{4}\right)}(\mathrm{SO}(7))\right](t ; \boldsymbol{x} ; \boldsymbol{n})=t^{\frac{1}{2}\left(5 n_{1}+3 n_{2}+n_{3}\right)} K_{\left(2,1^{4}\right)}^{\mathrm{USp}(6)}(\boldsymbol{x} ; t) \Psi_{\mathrm{USp}(6)}^{\left(n_{1}, n_{2}, n_{3}\right)}\left(t, x_{1}, x_{2} ; t\right),
$$

where the notations are explained below:

- $\boldsymbol{x}=\left(x_{1}, x_{2}\right)$ are the fugacities of the global symmetry USp(4).

- $\boldsymbol{n}=\left(n_{1}, n_{2}, n_{3}\right)$ are the background fluxes for $\mathrm{SO}(7)$, with the restriction

$$
n_{1} \geq n_{2} \geq n_{3} \geq 0
$$

- The argument $\left(t, x_{1}, x_{2}\right)$ of the Hall-Littlewood polynomial is obtained from the decomposition of the fundamental representation of $\mathrm{USp}(6)$ into representations of $\mathrm{SU}(2) \times \mathrm{USp}(4):$

$$
\chi_{[1,0,0]}^{\mathrm{USp}(6)}(\boldsymbol{y})=\chi_{[1]}^{\mathrm{SU}(2)}\left(t^{1 / 2}\right)+\chi_{[1,0]}^{\mathrm{USp}(4)}(\boldsymbol{x})
$$

so that $\boldsymbol{y}=\left(y_{1}, y_{2}, y_{3}\right)=\left(t^{1 / 2}, x_{1}, x_{2}\right)$.

- The prefactor $K_{\left(2,1^{4}\right)}^{\mathrm{USp}(6)}(\boldsymbol{x} ; t)$ comes from the following decomposition of the adjoint representation of $\mathrm{USp}(6)$ :

$$
\chi_{[2,0,0]}^{\mathrm{USp}(6)}\left(t^{1 / 2}, x_{1}, x_{2}\right)=\chi_{[2,0]}^{\mathrm{USp}(4)}(\boldsymbol{x})+\chi_{[1,0]}^{\mathrm{USp}(4)}(\boldsymbol{x}) \chi_{[1]}^{\mathrm{SU}(2)}\left(t^{1 / 2}\right)+\chi_{[2]}^{\mathrm{SU}(2)}\left(t^{1 / 2}\right) .
$$

Hence, according to (2.27), the prefactor is given by

$$
K_{\left(2,1^{4}\right)}^{\mathrm{USp}(6)}(\boldsymbol{x} ; t)=\mathrm{PE}\left[t \chi_{[2,0]}^{\mathrm{USp}(4)}(\boldsymbol{x})+t^{3 / 2} \chi_{[1,0]}^{\mathrm{USp}(4)}(\boldsymbol{x})+t^{2}\right] .
$$

For reference, we provide the Hilbert series with vanishing background fluxes:

$$
H\left[T_{\left(2,1^{4}\right)}(\mathrm{SO}(7))\right](t ; \boldsymbol{x} ; \mathbf{0})=\mathrm{PE}\left[\chi_{[2,0]}^{\mathrm{USp}(4)}(\boldsymbol{x}) t+\chi_{[1,0]}^{\mathrm{USp}(4)}(\boldsymbol{x}) t^{3 / 2}-t^{4}-t^{6}\right] .
$$

Hence the Coulomb branch of this theory is a complete intersection space with 12 complex dimensions, as expected from the quiver diagram (A.3). 


\section{A.1 The Coulomb branch Hilbert series of mirror theories}

Let us consider a Sicilian theory associated with a Riemann surface with genus $g$ and two sets of punctures: $2 m$ twisted $D_{N}$ punctures $\widetilde{\boldsymbol{\rho}}_{1}, \widetilde{\boldsymbol{\rho}}_{2}, \ldots, \widetilde{\boldsymbol{\rho}}_{2 m}$ and $n$ untwisted $D_{N}$ puncture $\rho_{1}, \rho_{2}, \ldots, \rho_{n}$.

Following the prescription of [6], the mirror of this Sicilian theory can be constructed by gluing $T_{\widetilde{\boldsymbol{\rho}}_{1}}\left(B_{N-1}\right), \ldots T_{\widetilde{\boldsymbol{\rho}}_{2 m}}\left(B_{N-1}\right)$ together with $T_{\boldsymbol{\rho}_{1}}\left(D_{N}\right), \ldots T_{\boldsymbol{\rho}_{n}}\left(D_{N}\right)$, with the common global symmetry group $B_{N-1}=\mathrm{SO}(2 N-1)$, which is a subgroup of $D_{N}=\mathrm{SO}(2 N)$, being gauged. The mirror quiver also contains $m+g-1$ hypermultiplets in the vector representation of the common gauge group $\mathrm{SO}(2 N-1)$. We test the prescription of [6] in section A.1.3 below using the mirror of a theory associated with a genus zero surface and four $\mathrm{SO}(4)$ punctures.

The Coulomb branch Hilbert series of the resulting mirror theory is therefore

$$
\begin{aligned}
H( & \left.t, \widetilde{\boldsymbol{x}}_{1}, \ldots, \widetilde{\boldsymbol{x}}_{m}, \boldsymbol{x}_{1}, \ldots, \boldsymbol{x}_{n}\right) \\
= & \sum_{n_{1} \geq \cdots \geq n_{N-1} \geq 0} t^{(g-1) \delta_{\mathrm{SO}(2 N-1)}(\boldsymbol{n})} t^{(m+g-1) \sum_{i=1}^{N-1} n_{i}} P_{\mathrm{SO}(2 N-1)}\left(t ; n_{1}, \ldots, n_{N-1}\right) \times \\
& \prod_{i=1}^{m} H\left[T_{\widetilde{\boldsymbol{\rho}}_{i}}\left(B_{N-1}\right)\right]\left(t ; \widetilde{\boldsymbol{x}}_{i} ; n_{1}, \ldots, n_{N-1}\right) \prod_{j=1}^{n} H\left[T_{\boldsymbol{\rho}_{j}}\left(D_{N}\right)\right]\left(t ; \boldsymbol{x}_{j} ; n_{1}, \ldots, n_{N-1}, 0\right),
\end{aligned}
$$

where the Casimir factor $P_{\mathrm{SO}(2 N-1)}$ is given by (A.9) and (A.6) of [1] and $\delta_{\mathrm{SO}(2 N-1)}$ is given by (2.24); the fugacities $\widetilde{\boldsymbol{x}}_{1}, \ldots, \widetilde{\boldsymbol{x}}_{m}$ correspond to the global symmetries associated with the twisted punctures $\widetilde{\boldsymbol{\rho}}_{1}, \ldots, \widetilde{\boldsymbol{\rho}}_{m}$ respectively, and similarly for the non-tilde fugacities. Here the factor denoted in blue is the contribution from the extra $m+g-1$ hypermultiplets in the vector representation of the gauge group $\mathrm{SO}(2 N-1)$.

It can be checked that formula (A.10) agrees with formula (4.10) of [10] and formula (2.8) of [31] for the HL index of the Sicilian theory in the case of two twisted punctures and genus 0 .

Below we demonstrate formula (A.10) using examples with $\mathrm{SO}(8)$ twisted and untwisted punctures on a Riemann surface with genus 0 .

\section{A.1.1 Twisted punctures $\left(2,1^{4}\right),\left(2,1^{4}\right)$ and untwisted puncture $(4,4)$}

Let us present an explicit example with $\widetilde{\boldsymbol{\rho}}_{1}=\widetilde{\boldsymbol{\rho}}_{2}=\left(2,1^{4}\right)$ and $\boldsymbol{\rho}_{1}=(4,4)$. The Coulomb branch Hilbert series of $T_{(4,4)}(\mathrm{SO}(8))$ is discussed in detail in appendix C.2 of [3]. From (A.10), the Hilbert series of the mirror of the Sicilian theory in question is

$$
\begin{aligned}
& H(t, \boldsymbol{a}, \boldsymbol{b}, c) \\
& \quad=\sum_{\substack{n_{1} \geq n_{2} \geq n_{3} \geq 0 \\
H\left[T_{\left(2,1^{4}\right)}(\mathrm{SO}(7))\right]\left(t ; b_{1}, b_{2} ; n_{1}, n_{2}, n_{3}\right) H\left[T_{(4,4)}(\mathrm{SO}(8))\right]\left(t ; c ; n_{1}, n_{2}, n_{3}, 0\right),}} t^{-\left(5 n_{1}+3 n_{2}+n_{3}\right)} P_{\mathrm{SO}(7)}\left(t ; n_{1}, n_{2}, n_{3}\right) H\left[T_{\left(2,1^{4}\right)}(\mathrm{SO}(7))\right]\left(t ; a_{1}, a_{2} ; n_{1}, n_{2}, n_{3}\right) \times \\
& \quad \text { (A. } 11)
\end{aligned}
$$

where the explicit expressions for $H\left[T_{\left(2,1^{4}\right)}(\mathrm{SO}(7))\right]$ and $H\left[T_{(4,4)}(\mathrm{SO}(8))\right]$ are given by (A.4) and by (C.18) of [3], respectively. The fugacities $\boldsymbol{a}=\left(a_{1}, a_{2}\right), \boldsymbol{b}=\left(b_{1}, b_{2}\right)$ and $c$ correspond to the global symmetries $\mathrm{USp}(4), \mathrm{USp}(4)$ and $\mathrm{USp}(2)$ respectively. 
The Higgs branch of the four dimensional Sicilian theory with the same punctures as this example was discussed on page 30 of [31] and Fixture 16 on page 35 of the same reference. Upon expanding (A.11) in a power series in $t$, we find an agreement with [31], namely

$$
H(t ; \boldsymbol{a}, \boldsymbol{b}, c)=\mathrm{PE}\left[t^{1 / 2} \chi_{[1]}^{\mathrm{SU}(2)}(c)\right] \times \widetilde{H}(t, \boldsymbol{a}, \boldsymbol{b}, c),
$$

where the first factor with the PE denotes the free hypermultiplet whose chiral multiplets transforming as a doublet of $\mathrm{SU}(2)$, and the first few terms in irreducible part $\widetilde{H}(t, \boldsymbol{a}, \boldsymbol{b}, c)$ are

$$
\begin{aligned}
\widetilde{H}(t ; \boldsymbol{a}, \boldsymbol{b}, c)= & 1+\chi_{[2,0,0,0,0]}^{C_{5}}(\boldsymbol{y}) t+\chi_{[0,0,0,0,1]}^{C_{5}}(\boldsymbol{y}) t^{3 / 2}+ \\
& \left(\chi_{[4,0,0,0,0]}^{C_{5}}(\boldsymbol{y})+\chi_{[0,2,0,0,0]}^{C_{5}}(\boldsymbol{y})+1\right) t^{2}+\ldots,
\end{aligned}
$$

where $\boldsymbol{y}=\left(y_{1}, \ldots, y_{5}\right)$ are fugacities of $\operatorname{USp}(10)$ and a possible fugacity map between $\boldsymbol{y}$ and $\boldsymbol{a}, \boldsymbol{b}, c$ is

$$
y_{1}=a_{1}, \quad y_{2}=a_{2}, \quad y_{3}=c, \quad y_{4}=b_{1}, \quad y_{5}=b_{2} .
$$

The plethystic logarithm of (A.13) indicates that there are 55 generators at order $t$ transforming in the representation $[2,0,0,0,0]$ of $\operatorname{USp}(10)$ and 132 generators at order $t^{3 / 2}$ in the representation $[0,0,0,0,1]$ of $\operatorname{USp}(10)$.

The unrefined Hilbert series $\widetilde{H}(t ; \boldsymbol{a}=\mathbf{1}, \boldsymbol{b}=\mathbf{1}, c=1)$ can be computed exactly:

$$
\begin{aligned}
\widetilde{H}(t ; & \boldsymbol{a}=\mathbf{1}, \boldsymbol{b}=\mathbf{1}, c=1)= \\
& \frac{1}{(1-t)^{32}(1+t)^{18}\left(1+t+t^{2}\right)^{16}} \times \\
& \left(1+2 t+40 t^{2}+194 t^{3}+1007 t^{4}+4704 t^{5}+18683 t^{6}+67030 t^{7}+220700 t^{8}\right. \\
& +657352 t^{9}+1796735 t^{10}+4540442 t^{11}+10610604 t^{12}+23011366 t^{13} \\
& +46535540 t^{14}+87887734 t^{15}+155277056 t^{16}+257288236 t^{17} \\
& +400453203 t^{18}+585971786 t^{19}+807195575 t^{20}+1047954388 t^{21} \\
& +1282842123 t^{22}+1481462886 t^{23}+1615002952 t^{24}+1662191888 t^{25} \\
& \left.+1615002952 t^{26}+\text { palindrome up to } t^{50}\right) .
\end{aligned}
$$

The irreducible component of the Coulomb branch is 16 quaternionic dimensional, as indicated by half of order of the pole at $t=1$ in the unrefined Hilbert series. Taking into account the free hypermultiplet, the Coulomb branch of this mirror theory is 17 quaternionic dimensional. This agrees with the result stated in the second bullet point on page 50 of [31] that the difference between the effective numbers of hypermultiplets and vector multiplets is $35-18=17$. 


\section{A.1.2 Twisted punctures $(6),\left(1^{6}\right)$ and untwisted puncture $\left(1^{8}\right)$}

From (A.10), the Hilbert series of the mirror of the Sicilian theory in question is

$$
\begin{aligned}
& H(t ; \boldsymbol{b}, \boldsymbol{c}) \\
& \quad=\sum_{\substack{n_{1} \geq n_{2} \geq n_{3} \geq 0 \\
H\left[T_{\left(1^{6}\right)}(\mathrm{SO}(7))\right]\left(t ; \boldsymbol{b} ; n_{1}, n_{2}, n_{3}\right) H\left[T_{\left(1^{8}\right)}(\mathrm{SO}(8))\right]\left(t ; \boldsymbol{c} ; n_{1}, n_{2}, n_{3}, 0\right),}} t^{-\left(5 n_{1}+3 n_{2}+n_{3}\right)} P_{\mathrm{SO}(7)}\left(t ; n_{1}, n_{2}, n_{3}\right) H\left[T_{(6)}(\mathrm{SO}(7))\right]\left(t ; n_{1}, n_{2}, n_{3}\right) \times \\
& \quad
\end{aligned}
$$

where $\boldsymbol{b}=\left(b_{1}, b_{2}, b_{3}\right)$ are fugacities for $\operatorname{USp}(6)$ and $\boldsymbol{c}=\left(c_{1}, \ldots, c_{4}\right)$ are fugacities for $\mathrm{SO}(8)$. Here the Hilbert series of the building blocks are given by

$$
\begin{aligned}
H\left[T_{(6)}(\mathrm{SO}(7))\right]\left(t ; n_{1}, n_{2}, n_{3}\right)= & t^{\frac{1}{2}\left(5 n_{1}+3 n_{2}+n_{3}\right)} \mathrm{PE}\left[t^{2}+t^{4}+t^{6}\right] \\
& \cdot \Psi_{\mathrm{USp}(6)}^{\left(n_{1}, n_{2}, n_{3}\right)}\left(t^{1 / 2}, t^{3 / 2}, t^{5 / 2} ; t\right) \\
= & 1, \\
H\left[T_{\left(1^{6}\right)}(\mathrm{SO}(7))\right]\left(t ; \boldsymbol{b} ; n_{1}, n_{2}, n_{3}\right)= & t^{\frac{1}{2}\left(5 n_{1}+3 n_{2}+n_{3}\right)} \mathrm{PE}\left[t \chi_{[2,0,0]}^{\mathrm{USp}(6)}(\boldsymbol{b})\right] \Psi_{\mathrm{USp}(6)}^{\left(n_{1}, n_{2}, n_{3}\right)}(\boldsymbol{b} ; t), \\
H\left[T_{\left(1^{8}\right)}(\mathrm{SO}(8))\right]\left(t ; \boldsymbol{c} ; n_{1}, n_{2}, n_{3}, n_{4}\right)= & t^{\frac{1}{2}\left(6 n_{1}+4 n_{2}+2 n_{3}\right)} \mathrm{PE}\left[t \chi_{[0,1,0,0]}^{\mathrm{SO}(8)}(\boldsymbol{b})\right] \Psi_{\mathrm{SO}(8)}^{\left(n_{1}, n_{2}, n_{3}, n_{4}\right)}(\boldsymbol{c} ; t) .
\end{aligned}
$$

It can be checked that (A.16) is equal to the Hilbert series of 48 free half-hypermultiplets:

$$
H(t ; \boldsymbol{b}, \boldsymbol{c})=\mathrm{PE}\left[t^{1 / 2} \chi_{[1,0,0]}^{\mathrm{USp}(6)}(\boldsymbol{b}) \chi_{[1,0,0,0]}^{\mathrm{SO}(8)}(\boldsymbol{c})\right] .
$$

This confirms the free field fixture \#8 on page 37 of [31].

\section{A.1.3 Four $\mathrm{SO}(4)$ twisted punctures: (2), (2), $\left(1^{2}\right),\left(1^{2}\right)$}

The aim of this example is to test the prescription of adding extra fundamental hypermultiplets of $\mathrm{SO}(2 N-1)$, as discussed in [6]. From (A.10), the Coulomb branch Hilbert series of the mirror of the $D_{4}$ Sicilian theory with twisted punctures $(2),(2),\left(1^{2}\right),\left(1^{2}\right)$ is

$$
\begin{aligned}
& H\left(t ; x_{1}, x_{2}\right) \\
& \quad=\sum_{n \geq 0} t^{-n} t^{n} P_{\mathrm{SO}(3)}(t ; n)\left[H\left[T_{(2)}(\mathrm{SO}(3))\right](t ; n)\right]^{2} \prod_{i=1}^{2} H\left[T_{(1,1)}(\mathrm{SO}(3))\right]\left(t ; x_{i} ; n\right) \\
& \quad=\sum_{n \geq 0} t^{-n} t^{n} P_{\mathrm{SO}(3)}(t ; n) \prod_{i=1}^{2} H\left[T_{(1,1)}(\mathrm{SO}(3))\right]\left(t ; x_{i} ; n\right),
\end{aligned}
$$

where the blue factor denotes the contribution of the extra hypermultiplet. The explicit expressions of each Hilbert series in the summand are

$$
\begin{aligned}
H\left[T_{(1,1)}(\mathrm{SO}(3))\right](t ; x ; n) & =\frac{t^{n} x^{-n}\left(1-t^{2} x^{2}+t^{2} x^{2 n}-x^{2+2 n}\right)}{\left(1-x^{2}\right)\left(1-t^{2} x^{-2}\right)\left(1-t^{2} x^{2}\right)}, \\
H\left[T_{(2)}(\mathrm{SO}(3))\right](t ; n) & =1 .
\end{aligned}
$$

Performing the summation, we find that the above Hilbert series is equal to (D.14) of [3]. Setting $x_{1}=x_{2}=1$, we recover the unrefined Hilbert series written in (D.15) of [3]. This is indeed equal to the Higgs branch Hilbert series of the $\mathrm{SO}(4)$ gauge theory with 2 flavors, in accordance with section 4.1 of [31]. 
Open Access. This article is distributed under the terms of the Creative Commons Attribution License (CC-BY 4.0), which permits any use, distribution and reproduction in any medium, provided the original author(s) and source are credited.

\section{References}

[1] S. Cremonesi, A. Hanany and A. Zaffaroni, Monopole operators and Hilbert series of Coulomb branches of $3 d \mathcal{N}=4$ gauge theories, JHEP 01 (2014) 005 [arXiv:1309.2657] [INSPIRE].

[2] D. Gaiotto and E. Witten, S-duality of Boundary Conditions In $\mathcal{N}=4$ Super Yang-Mills Theory, Adv. Theor. Math. Phys. 13 (2009) 721 [arXiv:0807.3720] [INSPIRE].

[3] S. Cremonesi, A. Hanany, N. Mekareeya and A. Zaffaroni, Coulomb branch Hilbert series and Hall-Littlewood polynomials, arXiv:1403.0585 [INSPIRE].

[4] A. Gadde, L. Rastelli, S.S. Razamat and W. Yan, Gauge Theories and Macdonald Polynomials, Commun. Math. Phys. 319 (2013) 147 [arXiv:1110.3740] [InSPIRE].

[5] D. Gaiotto, N=2 dualities, JHEP 08 (2012) 034 [arXiv:0904.2715] [INSPIRE].

[6] F. Benini, Y. Tachikawa and D. Xie, Mirrors of 3d Sicilian theories, JHEP 09 (2010) 063 [arXiv: 1007.0992] [INSPIRE].

[7] T. Nishioka, Y. Tachikawa and M. Yamazaki, 3d Partition Function as Overlap of Wavefunctions, JHEP 08 (2011) 003 [arXiv: 1105.4390] [INSPIRE].

[8] P.C. Argyres, M.R. Plesser and N. Seiberg, The Moduli space of vacua of N=2 SUSY QCD and duality in $N=1$ SUSY QCD, Nucl. Phys. B 471 (1996) 159 [hep-th/9603042] [INSPIRE].

[9] D. Gaiotto and S.S. Razamat, Exceptional Indices, JHEP 05 (2012) 145 [arXiv:1203.5517] [INSPIRE].

[10] M. Lemos, W. Peelaers and L. Rastelli, The superconformal index of class $\mathcal{S}$ theories of type D, JHEP 05 (2014) 120 [arXiv:1212.1271] [INSPIRE].

[11] A. Hanany and N. Mekareeya, Tri-vertices and SU(2)'s, JHEP 02 (2011) 069 [arXiv: 1012.2119] [INSPIRE].

[12] S. Kim, The Complete superconformal index for $N=6$ Chern-Simons theory, Nucl. Phys. B 821 (2009) 241 [Erratum ibid. B 864 (2012) 884] [arXiv:0903.4172] [INSPIRE].

[13] Y. Imamura and S. Yokoyama, Index for three dimensional superconformal field theories with general R-charge assignments, JHEP 04 (2011) 007 [arXiv:1101.0557] [INSPIRE].

[14] C. Krattenthaler, V.P. Spiridonov and G.S. Vartanov, Superconformal indices of three-dimensional theories related by mirror symmetry, JHEP 06 (2011) 008 [arXiv: 1103.4075] [INSPIRE].

[15] S.S. Razamat and B. Willett, Down the rabbit hole with theories of class $S$, arXiv: 1403.6107 [INSPIRE].

[16] P. Goddard, J. Nuyts and D.I. Olive, Gauge Theories and Magnetic Charge, Nucl. Phys. B 125 (1977) 1 [INSPIRE].

[17] V. Borokhov, A. Kapustin and X.-k. Wu, Monopole operators and mirror symmetry in three-dimensions, JHEP 12 (2002) 044 [hep-th/0207074] [INSPIRE]. 
[18] M.K. Benna, I.R. Klebanov and T. Klose, Charges of Monopole Operators in Chern-Simons Yang-Mills Theory, JHEP 01 (2010) 110 [arXiv:0906.3008] [INSPIRE].

[19] D. Bashkirov and A. Kapustin, Supersymmetry enhancement by monopole operators, JHEP 05 (2011) 015 [arXiv: 1007.4861] [INSPIRE].

[20] A. Hanany and E. Witten, Type IIB superstrings, BPS monopoles and three-dimensional gauge dynamics, Nucl. Phys. B 492 (1997) 152 [hep-th/9611230] [InSPIRE].

[21] N. Mekareeya, J. Song and Y. Tachikawa, $2 d$ TQFT structure of the superconformal indices with outer-automorphism twists, JHEP 03 (2013) 171 [arXiv:1212.0545] [INSPIRE].

[22] O. Chacaltana, J. Distler and Y. Tachikawa, Nilpotent orbits and codimension-two defects of $6 d \mathcal{N}=(2,0)$ theories, Int. J. Mod. Phys. A 28 (2013) 1340006 [arXiv:1203.2930] [INSPIRE].

[23] F. Benini, Y. Tachikawa and B. Wecht, Sicilian gauge theories and $\mathcal{N}=1$ dualities, JHEP 01 (2010) 088 [arXiv: 0909.1327] [INSPIRE].

[24] A. Hanany and R. Kalveks, in preparation.

[25] B. Feng and A. Hanany, Mirror symmetry by O3-planes, JHEP 11 (2000) 033 [hep-th/0004092] [INSPIRE].

[26] O. Chacaltana and J. Distler, Tinkertoys for the $D_{N}$ series, JHEP 02 (2013) 110 [arXiv:1106.5410] [INSPIRE].

[27] O. Chacaltana and J. Distler, Tinkertoys for Gaiotto Duality, JHEP 11 (2010) 099 [arXiv: 1008.5203] [INSPIRE].

[28] A. Gadde, E. Pomoni, L. Rastelli and S.S. Razamat, S-duality and 2d Topological QFT, JHEP 03 (2010) 032 [arXiv:0910.2225] [INSPIRE].

[29] A. Gadde, L. Rastelli, S.S. Razamat and W. Yan, The 4d Superconformal Index from q-deformed $2 d$ Yang-Mills, Phys. Rev. Lett. 106 (2011) 241602 [arXiv:1104.3850] [InSPIRE].

[30] G.W. Moore and Y. Tachikawa, On 2d TQFTs whose values are holomorphic symplectic varieties, arXiv:1106.5698 [INSPIRE].

[31] O. Chacaltana, J. Distler and A. Trimm, Tinkertoys for the Twisted D-Series, arXiv:1309.2299 [INSPIRE]. 